\title{
Fronts and bumps in spatially extended Kuramoto networks
}

\author{
Carlo R. Laing ${ }^{1}$ \\ Institute of Information and Mathematical Sciences, \\ Massey University, Private Bag 102-904 NSMC, \\ Auckland, New Zealand \\ ph: +64-9-4140800, extn 41038; fax: +64-9-441 8136
}

\begin{abstract}
We consider moving fronts and stationary "bumps" in networks of non-locally coupled phase oscillators. Fronts connect regions of high local synchrony with regions of complete asynchrony, while bumps consist of spatially-localised regions of partially-synchronous oscillators surrounded by complete asynchrony. Using the Ott-Antonsen ansatz we derive non-local differential equations which describe the network dynamics in the continuum limit. Front and bump solutions of these equations are studied by either "freezing" them in a travelling coordinate frame or analysing them as homoclinic or heteroclinic orbits. Numerical continuation is used to determine parameter regions in which such solutions exist and are stable.
\end{abstract}

Key words: Kuramoto, phase oscillators, synchrony, non-local, bifurcation, fronts.

\section{Introduction}

Networks of coupled oscillators have been studied for many years, often with an emphasis on the conditions under which some or all of the oscillators synchronize [1, 2, 3]. Among the different types of oscillators and couplings one could choose, the phase oscillator, coupled through a sinusoidal function of phase differences, is among the simplest $[4,5,6,7]$. The resulting "Kuramoto networks" are often studied when the coupling is all-to-all, i.e. there is no spatial structure to the network $[8,9,10,11]$, or (less often) when coupling is local $[12,13]$. However, nonlocal coupling arises naturally in several contexts $[14,15,16]$ and there has been recent interest in networks of non-locally coupled phase oscillators [17, 14, 18, 19]. One surprising phenomenon found in such systems is a "chimera" state, in which some fraction of the oscillators synchronize while the remainder are incoherent $[20,21,22,23,24,25,26,27,28]$. This splitting of the population into two groups is not a result of heterogeneity in the population, as it occurs in networks of identical oscillators, and has been shown to be robust with respect to parameter heterogeneity $[21,25]$. These chimera states were first analysed using a self-consistency argument due to Kuramoto [17, 14, 22, 23, 24, 29, 26, 20]. Later, the remarkable ansatz of Ott and Antonsen (OA) [8,30] was used to derive equations governing the macroscopic dynamics of chimera states $[25,21,27,28]$ and thus their stability could be determined.

From a macroscopic or statistical point of view, chimera states are stationary. In this paper we consider dynamics of Kuramoto networks which, from this point of view, are not stationary. Specifically, we concentrate mostly on moving fronts, which connect regions of high local synchrony with regions of complete asynchrony. We study several models, the first being that of [31], where oscillators are coupled (after a delay) to a non-local mean field. Rather than just showing the results of numerical simulation as these authors did, here we analyse fronts and stationary "bumps" in detail, finding parameter regimes for which they exist and are stable. These bump states consist of spatially-localised regions of partiallysynchronous oscillators in a background of asynchrony, and are studied here in both one and two spatial

\footnotetext{
${ }^{1}$ c.r.laing@massey.ac.nz 
dimensions. The second model is a spatially-extended version of that studied by [32], where oscillators are coupled to a non-local mean field, but with randomly chosen positive or negative coupling. For this system we find fronts with positive, negative and zero velocities, depending on parameter values.

The main technique we use is "freezing" [33,34] in which we move to a travelling coordinate frame in which fronts are stationary, and thus - after discretisation - satisfy a large number of non-linear simultaneous algebraic equations. We also demonstrate that fronts and bumps can be regarded as heteroclinic and homoclinic solutions, respectively, of low-dimensional systems of ordinary differential equations. In Sec. 2 we present the first model, and show results from our analysis of it in Sec. 3. The second model is presented in Sec. 4, with results shown in Sec. 5. We conclude in Sec. 6.

\section{Model 1: discrete and continuous}

Our first model consists of a network of phase oscillators driven by a delayed non-local mean field. The non-local mean field is defined through a weighted sum over all oscillators, and thus all oscillators are coupled to one another. As mentioned above, non-local coupling arises in several contexts, as do delays in the influence of oscillators on one another [35,24], and it is natural to consider both effects jointly.

\subsection{Spatially discrete model}

Consider $N$ phase oscillators equally spaced on the one dimensional spatial domain $[0, L]$. The state of oscillator $i$ at time $t$ is $\theta_{i}(t)$. The strength of coupling from oscillator $j$ to oscillator $i$ is $K_{i j}$ where

$$
K_{i j}=K(|i-j| \Delta x), \quad K(x)=(1 / 2) e^{-|x|}
$$

and $\Delta x=L /(N-1)$. We define the nonlocal mean field (which can be thought of as an order parameter which depends on space and time $[22,23,21]), R_{m}(t)$, by

$$
R_{m}(t)=\frac{L}{N} \sum_{j=1}^{N} K_{m j} e^{i \theta_{j}(t)}
$$

where $m=1, \ldots, N$. Since $K_{i j}$ decays with increasing $|i-j|$ the magnitude of $R_{m}(t)$ can be thought of as a measure of the level of synchronisation of oscillators near position $m$ at time $t$. In many previously studied examples, the dynamics of $\theta_{i}$ are determined by the current values of both $\theta_{i}$ and $R_{i}[24,26,20]$. However, here we use the delayed value of $R_{i}$ rather than its current value. Thus we introduce another set of variables $z_{m}(t)(m \in\{1, \ldots N\})$ defined by

$$
z_{m}(t)=\int_{0}^{\infty} R_{m}(t-\tau) h(\tau) d \tau
$$

where $h(\tau)$ is the probability density function of the delay $\tau$. If $h(\tau)=\delta(\tau-T)$, we have $z_{m}(t)=R_{m}(t-$ $T$ ), i.e. a discrete delay of size $T$. However, we choose $h(\tau)=e^{-\tau}$, which enables us to rewrite (3) as

$$
\frac{d z_{m}(t)}{d t}=R_{m}(t)-z_{m}(t)
$$

Lee et al. [31] considered a more general family of densities $h(\tau)$, but each one could be used to convert (3) into a set of ordinary differential equations involving $z_{m}$ and $R_{m}$. Writing $z_{j}=\rho_{j} e^{i \phi_{j}}$ we define our network dynamics by

$$
\frac{d \theta_{j}}{d t}=\omega_{j}+k \rho_{j} \sin \left(\phi_{j}-\theta_{j}\right) \quad j=1, \ldots N
$$

where $k$ is an overall coupling strength and the $\omega_{i}$ are chosen from some distribution with probability density function $g(\omega)$. Note that without the delay, i.e. if $h(\tau)=\delta(\tau)$, eqn. (5) is of the same form as that studied in $[17,23,22]$, although with possibly different coupling functions $K$, and an additional phase offset in the $\sin (\cdot)$ term. A typical example of the possible dynamics of (2),(4),(5) is shown in Fig. 1 when $g(\omega)$ is a Lorentzian with mean 5.5 and half-width-at-half-maximum 1. We see a wave of incoherence propagating with an approximately constant speed into a region of coherent oscillation, and this is the type of wave we would like to analyse. 

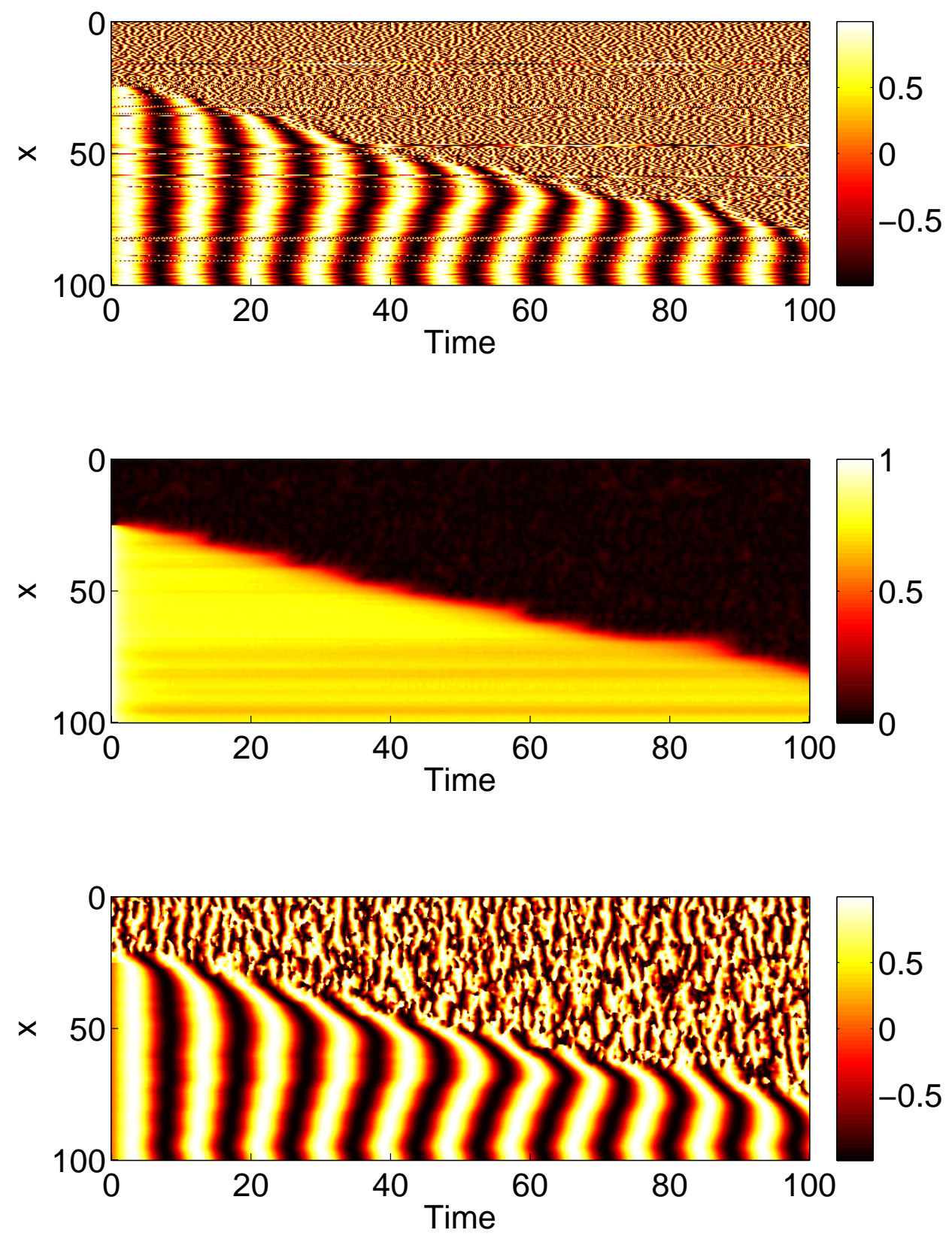

Figure 1: Typical dynamics of (2),(4),(5). Top: $\sin \theta_{i}$; middle: $\rho_{i}$; bottom: $\sin \phi_{i}$. Parameters: $k=12, L=100, N=5000$. 


\subsection{Continuum model}

To analyse the sort of behaviour seen in Fig. 1 we move to the continuum limit, i.e. we let $N \rightarrow$ $\infty$. Using $x$ as the spatial variable the system is then described by the probability density function $F(x, \omega, \theta, t)$ (the probability that an oscillator at position $x$ has intrinsic frequency $\omega$ and state $\theta$, at time $t$ ), and the dynamics of the network is

$$
\begin{aligned}
\frac{\partial \theta}{\partial t} & =\omega+k v(\theta, x, t) \\
\frac{\partial z(x, t)}{\partial t} & =R(x, t)-z(x, t)
\end{aligned}
$$

where

$$
R(x, t)=\int_{0}^{L} K(x-y) \int_{-\infty}^{\infty} \int_{-\pi}^{\pi} F(y, \omega, \theta, t) e^{i \theta} d \theta d \omega d y
$$

and

$$
v(\theta, x, t) \equiv \operatorname{Im}\left[z(x, t) e^{-i \theta}\right]=\frac{1}{2 i}\left[z(x, t) e^{-i \theta}-\bar{z}(x, t) e^{i \theta}\right]
$$

where overline indicates complex conjugate. The dynamics of $F$ is given by

$$
\frac{\partial F}{\partial t}+\frac{\partial}{\partial \theta}\{F(\omega+k v)\}=0
$$

and it is the closed system (7),(8),(9) and (10) that we now consider. This set of nonlocal PDEs seems formidable, but similar equations have been studied previously $[21,32,9]$ and several results allow us to simplify them. First, we use the OA ansatz $[8,30]$ to write $F$ as a Fourier series in $\theta$ :

$$
F(x, \omega, \theta, t)=\frac{g(\omega)}{2 \pi}\left[1+\left(\sum_{n=1}^{\infty}\{\alpha(x, \omega, t)\}^{n} e^{i n \theta}+\text { c.c. }\right)\right]
$$

where "c.c." indicates the complex conjugate of the previous term. This ansatz has been widely used; see $[9,10,25,21,27,28,35]$ for other examples. Substituting (11) into (8) and (10) we obtain

$$
R(x, t)=\int_{0}^{L} K(x-y) \int_{-\infty}^{\infty} \bar{\alpha}(y, \omega, t) g(\omega) d \omega d y
$$

and

$$
\frac{\partial \alpha(x, \omega, t)}{\partial t}=-i \omega \alpha(x, \omega, t)+(k / 2)\left[\bar{z}(x, t)-z(x, t) \alpha^{2}(x, \omega, t)\right]
$$

respectively. Choosing the distribution of intrinsic frequencies $g(\omega)$ to be

$$
g(\omega)=\frac{1 / \pi}{\left(\omega-\omega_{0}\right)^{2}+1}
$$

i.e. a Lorentzian centred at $\omega=\omega_{0}$ with half-width-at-half-maximum 1 , the integral over $\omega$ in (12) can be performed and we obtain

$$
R(x, t)=\int_{0}^{L} K(x-y) \bar{\alpha}\left(y, \omega_{0}-i, t\right) d y
$$

Setting $\omega=\omega_{0}-i$ in (13) and defining $a(x, t)=\alpha\left(x, \omega_{0}-i, t\right)$ we have

$$
\frac{\partial a(x, t)}{\partial t}=-\left(1+i \omega_{0}\right) a(x, t)+(k / 2)\left[\bar{z}(x, t)-z(x, t) a^{2}(x, t)\right]
$$

and

$$
R(x, t)=\int_{0}^{L} K(x-y) \bar{a}(y, t) d y
$$



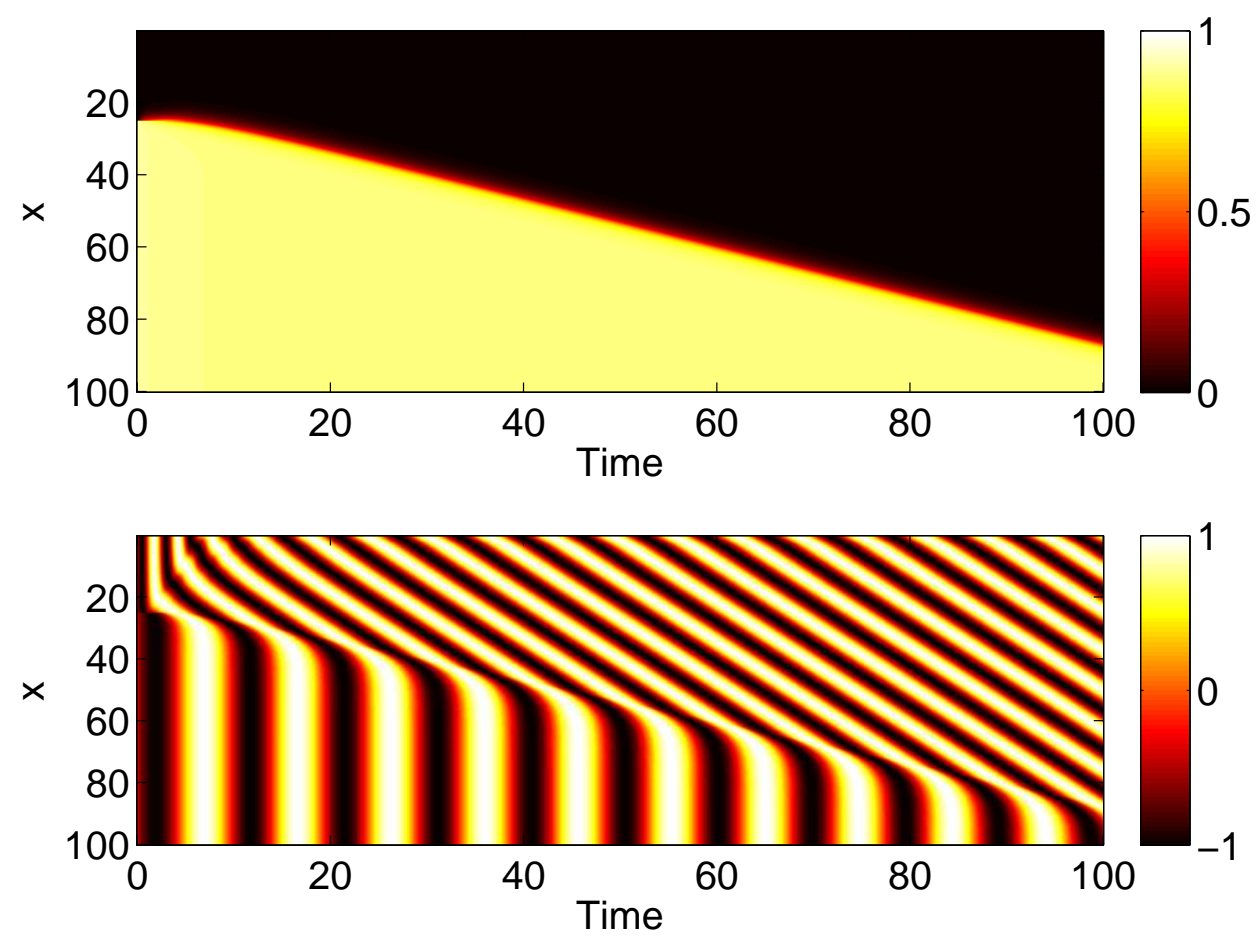

Figure 2: A solution of (19)-(21). Top: $|a(x, t)|$; bottom: $\sin (\arg (a(x, t)))$. Parameters: $k=12, L=100, \omega_{0}=5.5$. Compare with the lower two panels of Fig. 1.

Lastly, using the form of $K$, it can be shown by direct calculation that (17) can be rewritten as

$$
\left(1-\frac{\partial^{2}}{\partial x^{2}}\right) R(x, t)=\bar{a}(x, t)
$$

Thus we have the differential equations governing the macroscopic dynamics, valid in the limit $N \rightarrow \infty$ :

$$
\begin{aligned}
\frac{\partial a}{\partial t} & =-\left(1+i \omega_{0}\right) a+(k / 2)\left[\bar{z}-z a^{2}\right] \\
\frac{\partial z}{\partial t} & =R-z \\
\left(1-\frac{\partial^{2}}{\partial x^{2}}\right) R & =\bar{a}
\end{aligned}
$$

where all variables are evaluated at the same time and place. A solution of (19)-(21) for the same parameter values used in Fig. 1 is shown in Fig. 2, with good agreement in wave speeds. (Only $a$ is shown; the plots for $z$ look very similar, although $\arg (z)$ increases while $\arg (a)$ decreases.) In comparing Figs. 1 and 2 we see that the wave propagation in Fig. 2 is smooth, whereas that in Fig. 1 is less so. This is a result of the finite number of oscillators simulated in Fig. $1-$ using a larger number would make propagation smoother. Note that eqns. (19)-(21) are a particular version of the more general equations derived in [31]. We now proceed with a limited analysis of (19)-(21).

\section{Results}

\subsection{Spatially uniform states}

Firstly, consider spatially uniform states of (19)-(21). Clearly $z=a=0$ is a spatially uniform steady state of (19)-(21). Figure 2 suggests that there is another spatially uniform partially synchronous state, 


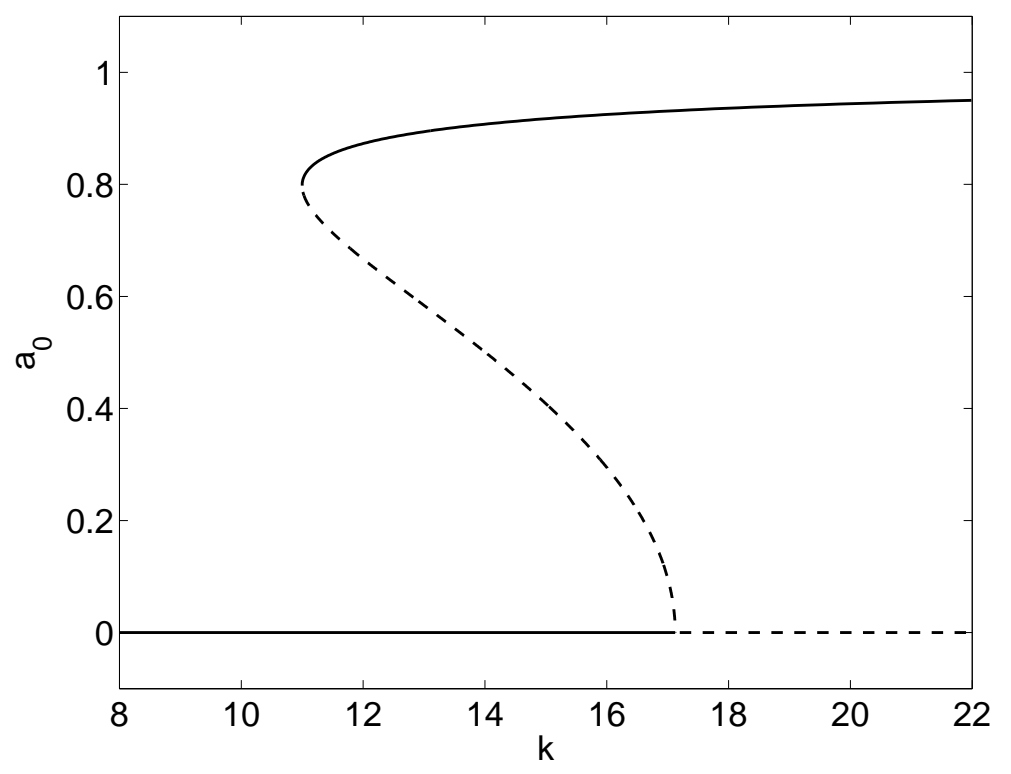

Figure 3: The amplitude of spatially uniform solutions of (19)-(21). Solid: stable, dashed: unstable. A saddle-node bifurcation occurs at $k=2 \omega_{0}$ and a pitchfork bifurcation at $k=\left(\omega_{0}^{2}+4\right) / 2$. Parameters: $\omega_{0}=5.5$.

for which $|a|$ is constant and the argument of $a$ varies linearly with time. Substituting $a=a_{0} e^{i \Omega t}$ where $a_{0}$ and $\Omega$ are real and $a_{0}>0$ into (19)-(21) gives

$$
\left[1+i\left(\omega_{0}+\Omega\right)\right] a_{0}=\frac{a_{0} k}{2}\left[\frac{1}{1+i \Omega}-\frac{a_{0}^{2}}{1-i \Omega}\right]
$$

from which we can determine that

$$
k=\frac{\omega_{0}^{2}\left(1-a_{0}^{2}\right)^{2}+4}{2\left(1-a_{0}^{2}\right)}
$$

and

$$
\Omega=-\sqrt{\frac{k\left(1-a_{0}^{2}\right)}{2}-1}
$$

To analyse solutions of (23) we note that setting $\partial k / \partial a_{0}=0$ (in order to find a saddle-node bifurcation of solutions with $a_{0}>0$ ) gives

$$
1-a_{0}^{2}=2 / \omega_{0}
$$

which can only be satisfied when $\omega_{0}>2$. Substituting (25) into (23) gives $k=2 \omega_{0}$ and thus for $\omega_{0}>2$ a pair of nontrivial spatially uniform states is created at $a_{0}=\sqrt{1-2 / \omega_{0}}$ as $k$ increases through $2 \omega_{0}$; see Fig. 3. Letting $a_{0} \rightarrow 0$ in (23) we see that the branch of nontrivial solutions with smaller $a_{0}$ dies at the origin at $k=\left(\omega_{0}^{2}+4\right) / 2$; again see Fig. 3. Thus for $2 \omega_{0}<k<\left(\omega_{0}^{2}+4\right) / 2$ and $\omega_{0}>2$ there are three spatially uniform states (two with non-zero values of $a_{0}$ ). If $\omega_{0}<2$ then one nontrivial spatially uniform state bifurcates from the origin as $k$ increases through $\left(\omega_{0}^{2}+4\right) / 2$.

A Turing analysis of the zero state (linearising the dynamics around it and assuming that $a \sim e^{\lambda t} e^{i \kappa x}$ and $z \sim e^{\bar{\lambda} t} e^{-i \kappa x}$ where $\lambda \in \mathbb{C}$ ) gives the relationship

$$
(\lambda+1)^{2}+i \omega_{0}(\lambda+1)-\frac{k}{2\left(1+\kappa^{2}\right)}=0
$$

from which it follows that the zero state is stable to all perturbations for $k<\left(\omega_{0}^{2}+4\right) / 2$ and unstable otherwise, with the instability at $k=\left(\omega_{0}^{2}+4\right) / 2$ corresponding to $\kappa=0$, i.e. the spatially uniform mode. 

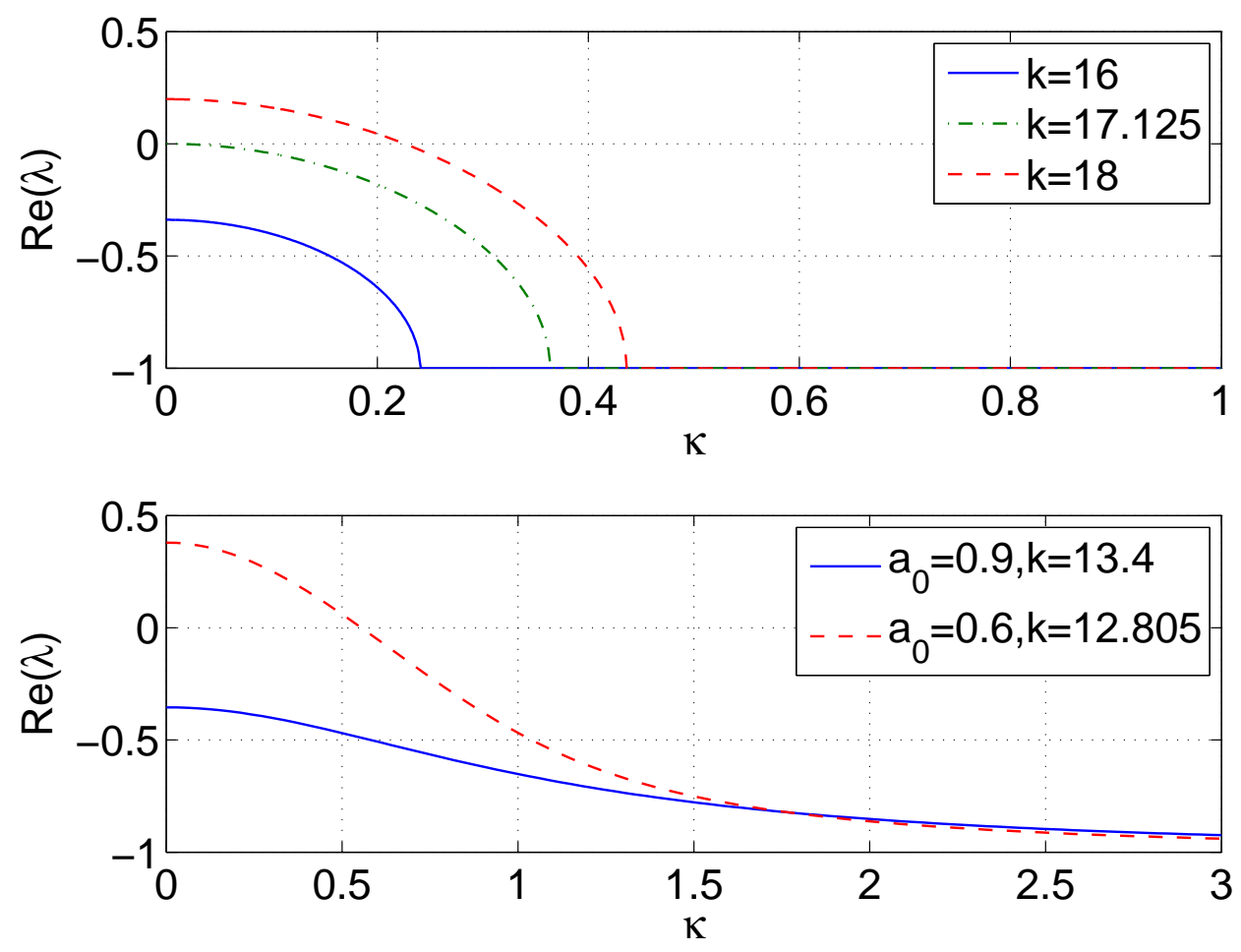

Figure 4: Top: $\operatorname{Re}(\lambda)$ as a function of $\kappa$ for the zero state, as determined from (26), for $k=16,17.125$ and 18 . The instability occurs when $k=\left(\omega_{0}^{2}+4\right) / 2=17.125$. Bottom: $\operatorname{Re}(\lambda)$ as a function of $\kappa$ for two points (one stable, one unstable) on the non-trivial branch of spatially-uniform solutions in Fig. 3, as determined from (27)-(28). Parameters: $\omega_{0}=5.5$.

This is demonstrated in Fig. 4 (top), where we plot $\operatorname{Re}(\lambda)$ as a function of $\kappa$ for three different values of $k$. Similarly, linearising about the nontrivial spatially uniform state we obtain

$$
\begin{array}{r}
{\left[2\left(1+\kappa^{2}\right)(1+\lambda-i \Omega)(\lambda+B)-k\right]} \\
\times\left[2\left(1+\kappa^{2}\right)(1+\bar{\lambda}-i \Omega)(\bar{\lambda}+B)-k\right]-k^{2} a_{0}^{4}=0
\end{array}
$$

where

$$
B=1-i\left(\Omega-\omega_{0}\right)+\frac{k a_{0}^{2}}{1-i \Omega}
$$

Analysing this expression shows that the solution with large $a_{0}$ is stable to all perturbations, and the solution with small $a_{0}$ is always unstable, when these solutions exist. Figure 4 (bottom) shows an example of these stabilities, where we plot $\operatorname{Re}(\lambda)$ as a function of $\kappa$ for two points on the branch of non-trivial solutions shown in Fig. 3. Combining these results we conclude that the system shows bistability for $2 \omega_{0}<k<\left(\omega_{0}^{2}+4\right) / 2$, and a typical arrangement of spatially uniform states is shown in Fig. 3. This result (when spatial variations and perturbations are not considered) was found in [35]. We thus conclude that for the spatial interactions considered here, their inclusion does not lead to new instabilities. When $\omega_{0}<2$, the nontrivial spatially uniform state is stable, when it exists.

\subsection{Fronts}

The presence of two coexisting stable spatially uniform states suggests that fronts connecting them may also exist, as we have already seen in Fig. 2. In order to study these fronts we first freeze them, by going to a coordinate frame moving at the same speed as them [33, 34], using the transformation $x \mapsto x-c t$, where $c$ is the speed of the front. From Fig. 2 it is clear that even if we were to go to a coordinate frame moving at the same speed as the wave, the variables $a$ and $z$ would still be rotating 
with fixed amplitude in the complex plane, at each spatial position. Thus to completely freeze the solutions we need to move to a coordinate frame in which both $a$ and $z$ are also rotating, but with opposite angular velocities, using the transformation $a \mapsto a e^{-i s t}, z \mapsto z e^{i s t}$ (and $R \mapsto R e^{i s t}$ ), where $-s$ is the angular velocity at which $a$ is rotating $(z$ and $R$ rotate with angular velocity $s)$. In such a coordinate frame, eqns. (19)-(21) become

$$
\begin{aligned}
\frac{\partial a}{\partial t} & =c \frac{\partial a}{\partial x}-\left[1+i\left(\omega_{0}+s\right)\right] a+(k / 2)\left[\bar{z}-z a^{2}\right] \\
\frac{\partial z}{\partial t} & =c \frac{\partial z}{\partial x}+R-(1-i s) z \\
\left(1-\frac{\partial^{2}}{\partial x^{2}}\right) R & =\bar{a}
\end{aligned}
$$

Even if we find a front which is a steady state of (29)-(31), this solution is not unique as we can translate it by an arbitrary amount in space, and also perform the rigid rotation $a \mapsto a e^{i \gamma}, z \mapsto z e^{-i \gamma}$ where $\gamma$ is a real constant, both of which give other steady states of (29)-(31). In order to remove these degeneracies (and find $c$ and $s$ ) we impose two "pinning" conditions on the solutions of (29)-(31). The first condition is

$$
\int_{0}^{L} \frac{d \widehat{u}}{d x}(|a(x, t)|-\widehat{u}) d x=0
$$

where $\widehat{u}(x)$ is a fixed "template" function. Equation (32) results from minimising the $L^{2}$ norm of the difference between $a(x, t)$ and $\widehat{u}(x)$ [34]. We will use

$$
\widehat{u}(x)=\frac{1+\tanh [0.2(x-L / 2)]}{2}
$$

i.e. an approximate front, centred at $x=L / 2$. The second condition is

$$
\operatorname{Im}(a(L, t))=0 .
$$

which removes the rotational invariance mentioned above. An example of numerically solving (29)(31) subject to (32) and (34) is shown in Fig. 5. The freezing is implemented at $t=43$, i.e. before that we set $s=c=0$ and conditions (32) and (34) are not imposed. We see an initially propagating front, with $\arg (a)$ varying linearly with time, then, once the front is frozen and transients have died out, all variables are stationary (the plots for $z$ are very similar). We find numerically that for these parameter values, $s=-0.65492$ and $c=0.68321$.

Having found a stable stationary solution of (29)-(31) subject to (32) and (34) by numerical integration, we can follow it as parameters are varied using pseudo-arclength continuation. In practice, we discretise $a(x), z(x)$ and $R(x)$ in space and solve the discretised versions of

$$
\begin{aligned}
& 0=c \frac{d a}{d x}-\left[1+i\left(\omega_{0}+s\right)\right] a+(k / 2)\left[\bar{z}-z a^{2}\right] \\
& 0=c \frac{d z}{d x}+R-(1-i s) z \\
& 0=\bar{a}-\left(1-\frac{d^{2}}{d x^{2}}\right) R \\
& 0=\int_{0}^{L} \frac{d \widehat{u}}{d x}(|a|-\widehat{u}) d x \\
& 0=\operatorname{Im}(a(L))
\end{aligned}
$$

Spatial derivatives are approximated by second-order finite differences and all functions have zero first derivative at $x=0, L$. Using finer discretisations did not alter our results.

A result of such continuation is shown in Fig. 6. Stability of solutions can be determined by examining the eigenvalues of the appropriate part of the linearisation of (35)-(39) about a solution of (35)-(39). 

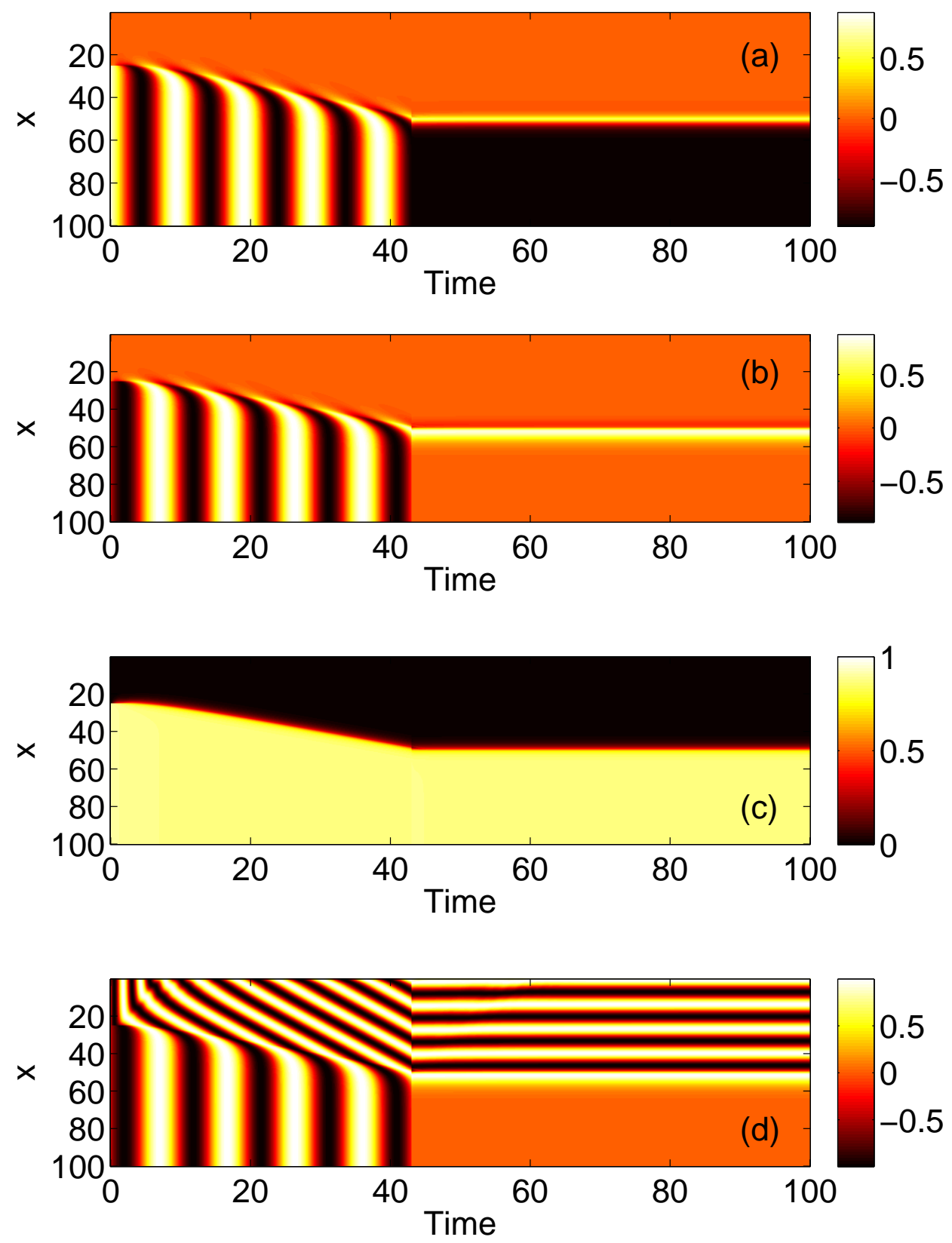

Figure 5: A solution of (29)-(31) subject to (32) and (34). The freezing is implemented at $t=43$. (a): $\operatorname{Re}(a) ;(\mathrm{b}): \operatorname{Im}(a) ;(\mathrm{c}):|a| ;(\mathrm{d})$ : $\sin (\arg (a))$. Parameters: $L=100, \omega_{0}=5.5, k=12$. 


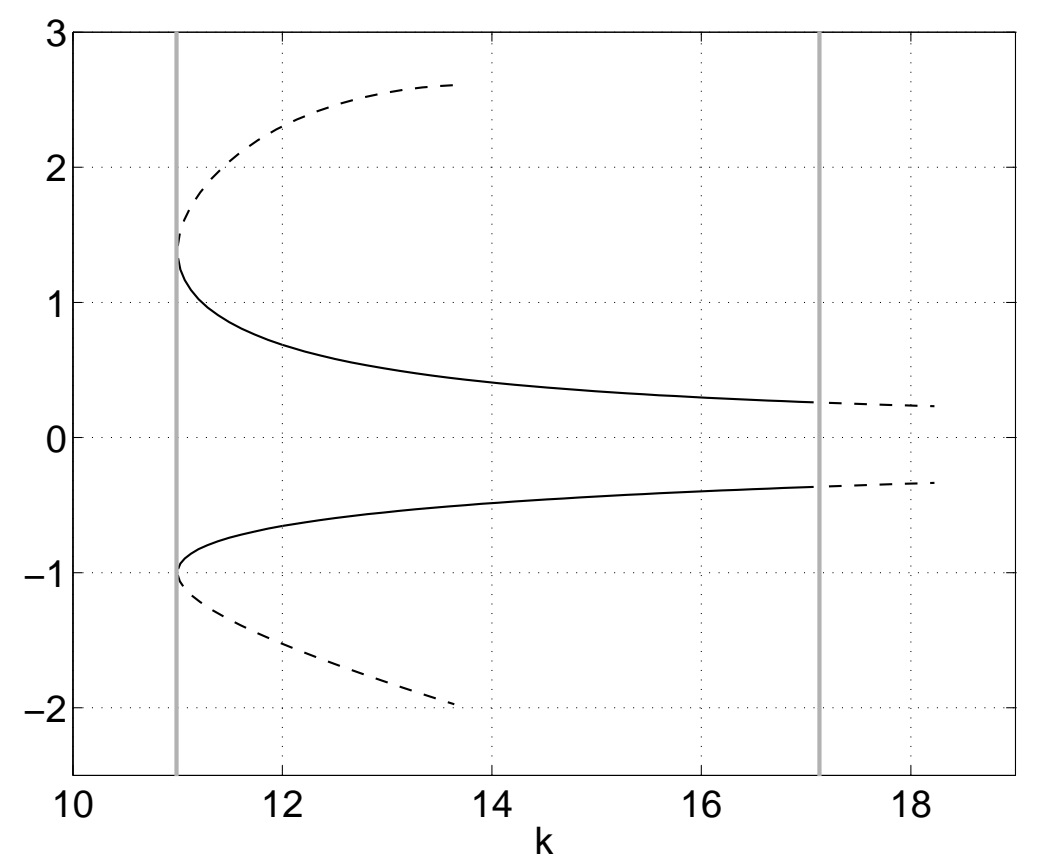

Figure 6: $c$ (positive) and $s$ (negative) as functions of $k$ for front solutions of (35)-(39). Solid curves: stable; dashed curves: unstable. The vertical grey lines mark $k=2 \omega_{0}$ (left) and $k=\left(\omega_{0}^{2}+4\right) / 2$ (right). Parameters: $L=100, \omega_{0}=5.5$.

We see that as $k$ is decreased, the front is destroyed in a saddle-node bifurcation with an unstable front. Recalling from Sec. 3.1 that nontrivial spatially uniform states only exist when $2 \omega_{0}<k$ (for $2<\omega_{0}$ ), we see that this saddle-node bifurcation is just that of these nontrivial spatially uniform states. Similarly, the bifurcation that occurs as $k$ is increased is caused by the zero state becoming unstable at $k=\left(\omega_{0}^{2}+4\right) / 2$. Following fronts for other parameter values we find a straight-forward result: stable fronts exist when the spatially-uniform system is bistable.

\subsection{Bumps}

\subsubsection{One spatial dimension}

Another phenomenon observed by [31] was the existence of "bridges", localised regions of coherent oscillators, formed when counterpropagating fronts met. An example is shown in Fig. 7, both for the PDEs (19)-(21) and the network of oscillators governed by (2)-(5). Note that for fixed parameters, only one stable "bump" of this form may exist; a variety of different initial conditions lead to the same attractor (up to an arbitrary shift in space). These bumps of coherence are in some sense the opposite of the chimera Ising walls found by [26], which consist of a localised region of incoherent oscillators separating two groups of coherent oscillators in anti-phase with one another. The analysis below strongly suggests that the coherent bumps shown in Fig. 7 exist on an infinite domain, and Kawamura states that his results were unchanged when he increased the system size [26]; thus the bumps shown here and Kawamura's chimera Ising walls are different from the chimeras studied by others [25, 21, 23, 22, 24, 28], all of whom used a finite domain with periodic boundary conditions.

In these bump solutions, the coherent oscillators rotate with a common frequency, and thus they are 

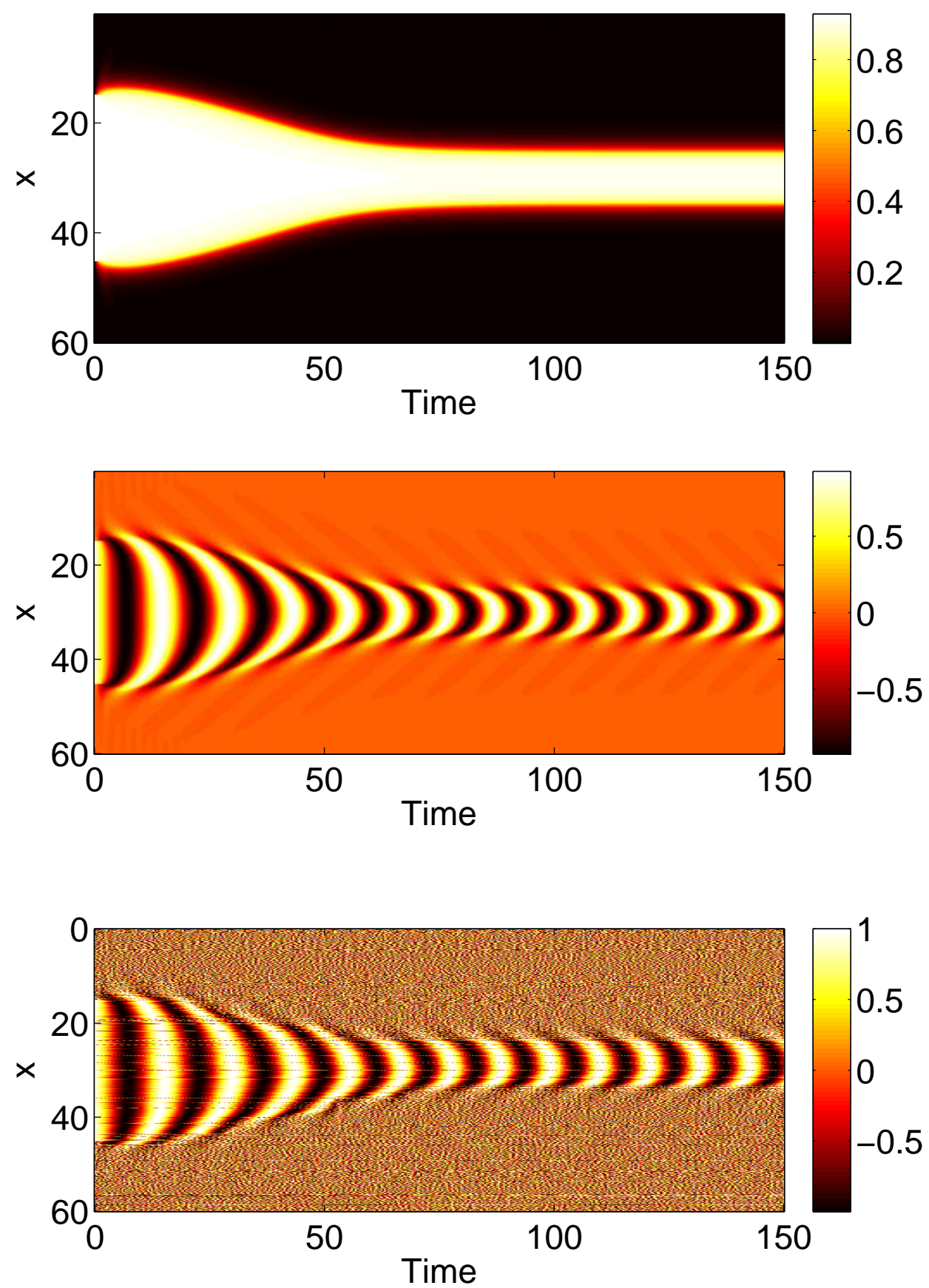

Figure 7: Top and middle: a solution of (19)-(21). Top: $|a(x, t)|$. Middle: $\operatorname{Re}(a(x, t))$. Bottom: a simulation of (2)-(5); $\cos \theta_{i}$ is shown color-coded. $N=15,000$ and only every 20th oscillator is shown. Other parameters: $L=60, \omega_{0}=5.5, k=16$. 


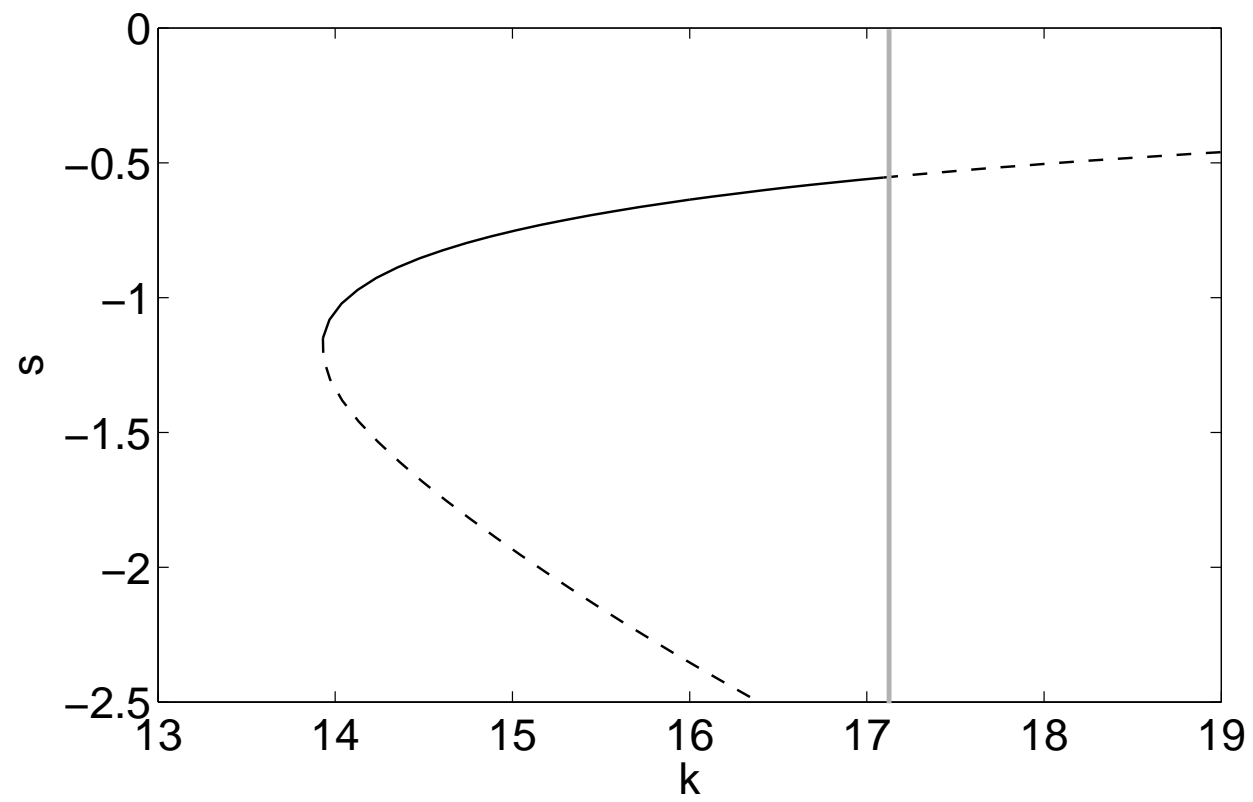

Figure 8: Angular velocity $s$ as a function of $k$ for stationary bump solutions of (40)-(42). Solid curve: stable; dashed curve: unstable. The vertical grey line is at $k=\left(\omega_{0}^{2}+4\right) / 2$. Other parameters: $L=60, \omega_{0}=5.5$.

stationary solutions of

$$
\begin{aligned}
\frac{\partial a}{\partial t} & =-\left[1+i\left(\omega_{0}+s\right)\right] a+(k / 2)\left[\bar{z}-z a^{2}\right] \\
\frac{\partial z}{\partial t} & =R-(1-i s) z \\
\left(1-\frac{\partial^{2}}{\partial x^{2}}\right) R & =\bar{a}
\end{aligned}
$$

where $s$ is this common angular frequency. To study bumps in the same way as fronts we need to specify the spatial position of a bump, which we do by requiring $a$ (and $z$ and $R$ ) to be even about $x=L / 2$ (i.e. centering the bump in the middle of the domain). We also append a scalar equation to (40)(42) in order to remove the rotational degeneracy and determine $s$, which we choose to be the equation $\operatorname{Im}(a(L / 2, t))=0$. In the same way as for the fronts, we can follow bumps as parameters are varied and determine their stability. Typical results are shown in Fig. 8. The instability at large $k$ is caused by the instability of the zero state, as seen in Fig. 3 of [31], but the saddle-node bifurcation at low $k$ is unrelated to the existence or otherwise of non-trivial spatially uniform states - see Fig. 6 . The saddlenode bifurcation seen in Fig. 8 can be followed as a second parameter is varied - see Fig. 9, where $\omega_{0}$ is varied. Stable fronts exist between the left- and right-most curves, whereas stable bumps only exist between the central and right-most curves.

Another way to view these bump solutions is to recognise that they are homoclinic orbits to the origin of the (complex) ODEs

$$
\begin{aligned}
& \frac{d R}{d x}=T \\
& \frac{d T}{d x}=R-\bar{a}
\end{aligned}
$$

where

$$
a=\frac{-\left[1+i\left(\omega_{0}+s\right)\right]+\sqrt{\left[1+i\left(\omega_{0}+s\right)\right]^{2}+k^{2}|R|^{2} /\left(1+s^{2}\right)}}{k R /(1-i s)}
$$




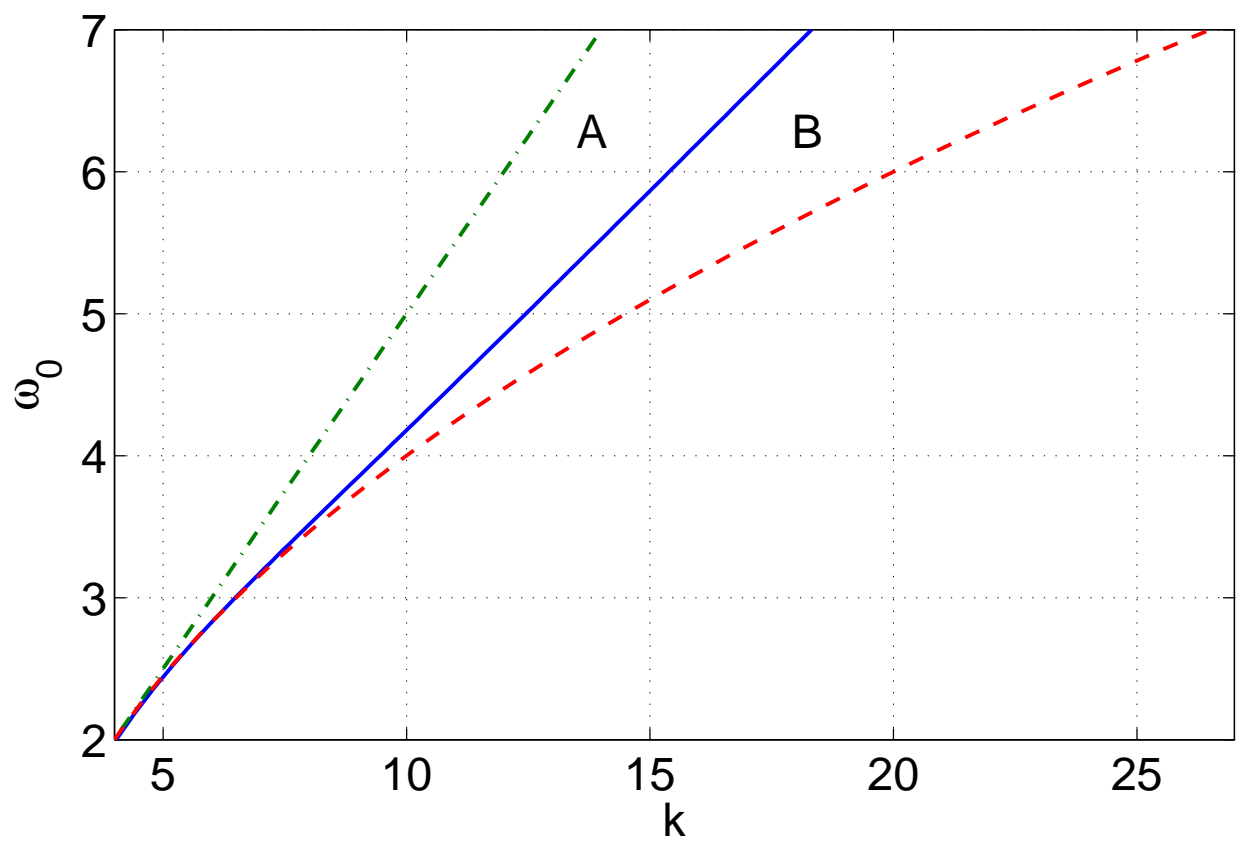

Figure 9: Solid blue: saddle-node bifurcation of bump solutions of (40)-(42). Green dash-dotted: $k=2 \omega_{0}$, to the left of which no nontrivial spatially uniform states exist. Red dashed: $k=\left(\omega_{0}^{2}+4\right) / 2$, to the right of which the zero state is unstable. Stable fronts exist in regions A and B, while stable bumps exist only in region B. Other parameters: $L=60$.

obtained by setting the time derivatives in (40) and (41) to zero and solving for $a$ in terms of $R$, and rewriting (42) as two first order equations. A number of points regarding (43)-(45) can be made:

- Having found a solution of (43)-(45) we can obtain another one by the transformation $(R, T) \mapsto$ $\left(R e^{i \gamma}, T e^{i \gamma}\right)$ for any real $\gamma$.

- This system is reversible [36] under the transformation $\rho_{1}:(x, T, R) \mapsto(-x,-T, R)$, and under the transformation $\rho_{2}:(x, T, R) \mapsto(-x, T,-R)$. We will only consider solutions which are symmetric under $\rho_{1}$.

- From our previous results, and consistent with the reversibility [36], homoclinic orbits to the origin are codimension zero, i.e. for any $k$ and $\omega_{0}$ between the solid and dashed curves in Fig. 9 we expect to find such an orbit.

Writing $R=R_{1}+i R_{2}$ and $T=T_{1}+i T_{2}$ we rewrite (43)-(44) as

$$
\begin{aligned}
& \frac{d R_{1}}{d x}=T_{1} \\
& \frac{d R_{2}}{d x}=T_{2} \\
& \frac{d T_{1}}{d x}=R_{1}-\operatorname{Re}(\bar{a}) \\
& \frac{d T_{2}}{d x}=R_{2}-\operatorname{Im}(\bar{a})
\end{aligned}
$$

The Jacobian of (46)-(49) at the origin is of the form

$$
J=\left(\begin{array}{cccc}
0 & 0 & 1 & 0 \\
0 & 0 & 0 & 1 \\
\alpha & \beta & 0 & 0 \\
-\beta & \alpha & 0 & 0
\end{array}\right)
$$




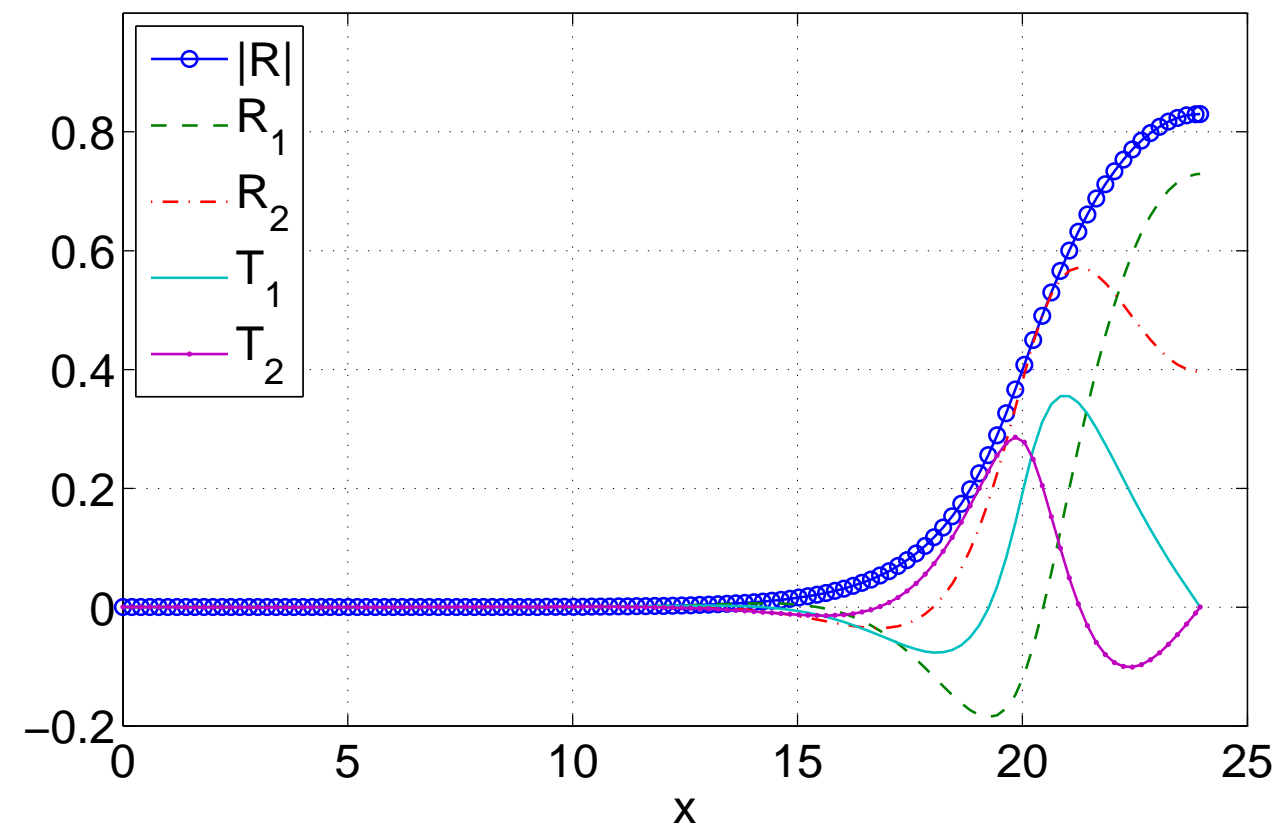

Figure 10: A solution of (46)-(49), with integration stopped when $T_{1}=T_{2}=0$. See text for initial conditions. For this orbit, $s=-0.75548$. Other parameters: $\omega_{0}=5.5, k=15, \epsilon=10^{-6}$.

where $\alpha$ and $\beta$ are real and generically not zero. Thus the eigenvalues of $J$ are given by $\lambda^{2}=\alpha \pm$ $i \beta$, i.e. the origin generically has a two-dimensional stable manifold and a two-dimensional unstable manifold, and homoclinic orbits spiral into and out from the origin.

The usual method of finding a homoclinic orbit to the origin for (46)-(49) would be to find the two normalised eigenvectors $v_{1}$ and $v_{2}$ of $J$ which span the unstable eigenspace, then set the initial condition for (46)-(49) to be

$$
\left(\begin{array}{l}
R_{1} \\
R_{2} \\
T_{1} \\
T_{2}
\end{array}\right)=\epsilon\left(v_{1} \cos \theta+v_{2} \sin \theta\right)
$$

for small $\epsilon$ and integrate (46)-(49), varying $\theta$ until a trajectory which returns to the origin is found. However, from the structure of $J$, varying $\theta$ can be shown to be equivalent to the transformation $(R, T) \mapsto\left(R e^{i \gamma}, T e^{i \gamma}\right)$ mentioned above. Thus we choose our initial condition to be $\epsilon v_{1}$, and integrate (46)-(49) until we hit the symmetry section $\operatorname{Fix}\left(\rho_{1}\right) \equiv\left\{(T, R): \rho_{1}(T, R)=(T, R)\right\}$. For fixed $k, \omega_{0}$ and initial condition, we find that (for a single bump solution) there is only one value of $s$ for which the solution hits the symmetry section, i.e. $T_{1}$ and $T_{2}$ are both zero at the same value of $x$. This is the value of $s$ associated with the bump. See Fig. 10 for an example. Having found this solution, the rest of the bump solution can be obtained using the reversibility of the system.

These homoclinic orbits can be followed as parameters such as $k$ are varied, where only a single value of $s$ needs to be found for each value of $k$. The results obtained agree with those in Figs. 8 and 9 (results not shown), but note that stability of solutions cannot be determined using this method. The theory of homoclinic orbits in reversible system is extensive [37, 36], and (46)-(49) could be analysed further (to find, for example, $N$-bump solutions $[36,38]$ ) but we will not do so here.

\subsubsection{2 spatial dimensions}

Lee et al. [31] also considered the equivalent of (6)-(9) but in two spatial dimensions. In this case, eqn. (8) is modified to involve an integral over two-dimensional space, and $K$ is chosen to be Green's 

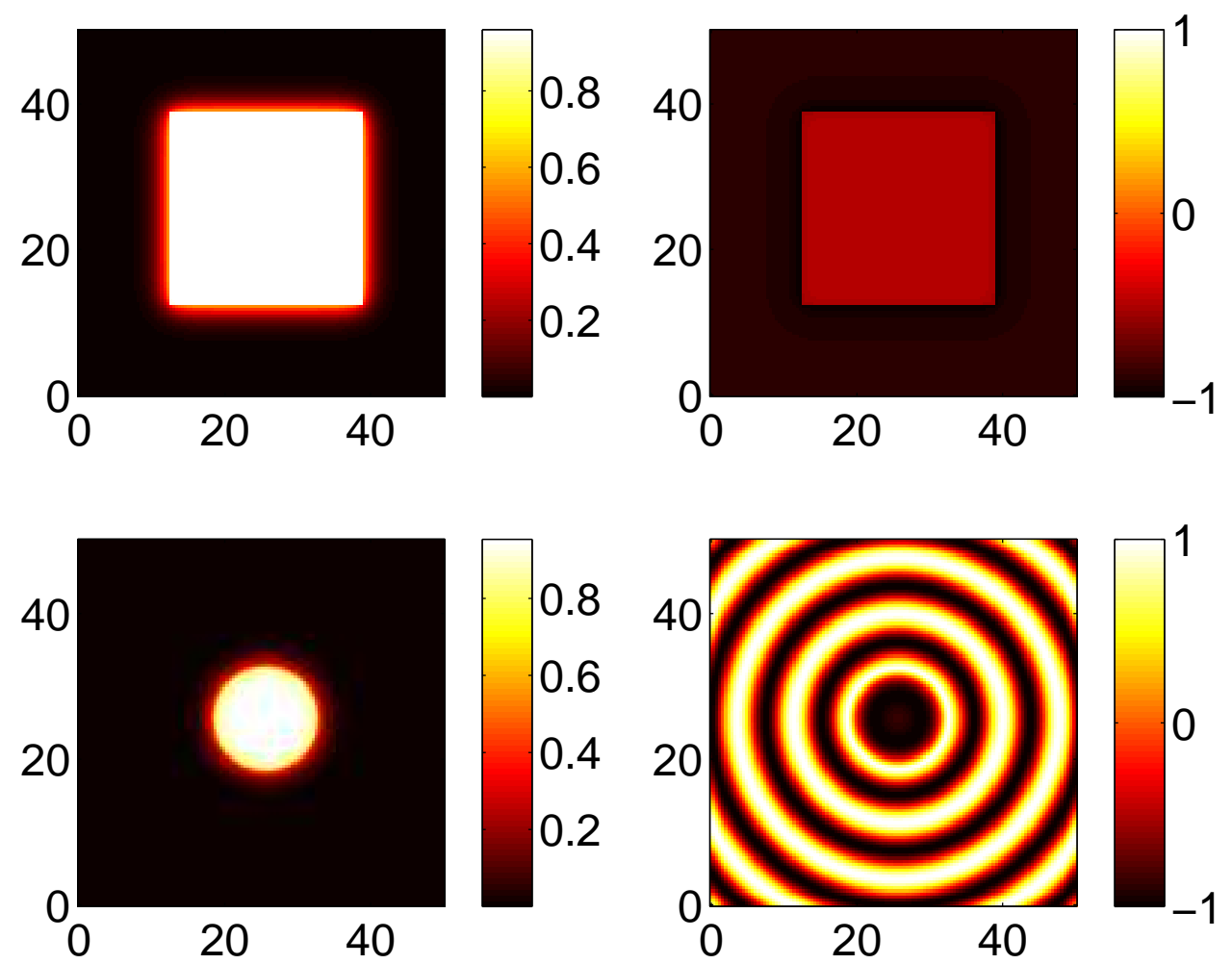

Figure 11: Solutions of (19),(20) and (53). Left column: $|a|$; right column: $\sin (\arg (a))$. Top row: $t=0.75$; bottom row: $t=300$. Domain is $50 \times 50$. Other parameters: $\omega_{0}=7.5, k=28$.

function of $1-\nabla^{2}$, i.e.

$$
K(x)=\frac{K_{0}(|x|)}{2 \pi}
$$

where $x \in \mathbb{R}^{2}$ and $K_{0}$ is the modified Bessel function of the second kind, of order 0. (In the onedimensional case, $K(x)=(1 / 2) e^{-|x|}$ was used, as it is the Green's function of $1-\partial^{2} / \partial x^{2}$.) Equations (19) and (20) remain unchanged, and (21) is replaced by

$$
\left(1-\nabla^{2}\right) R=\bar{a}
$$

In the same way as in one spatial dimension, in two dimensions we also have moving fronts which can meet to form spatially-localised patterns. Fig. 11 (bottom row) shows such a state in which $|a|$ is not changing over time, while the argument of $a$ at each spatial point varies linearly with time ( $z$ behaves similarly). As in one spatial dimension, the radius of a stable circular "bump" is determined by the parameter values and a variety of initial conditions lead to the same solution (up to an arbitrary translation in space).

To investigate these types of circularly-symmetric solutions it is natural to use polar coordinates in space, drop any angular dependence, and to move to a coordinate system in which $a$ and $z$ rotate at constant (and opposite) angular velocity, as above. Thus we consider

$$
\begin{aligned}
\frac{\partial a}{\partial t} & =-\left[1+i\left(\omega_{0}+s\right)\right] a+(k / 2)\left[\bar{z}-z a^{2}\right] \\
\frac{\partial z}{\partial t} & =R-(1-i s) z \\
\left(1-\frac{\partial^{2}}{\partial r^{2}}-\frac{1}{r} \frac{\partial}{\partial r}\right) R & =\bar{a}
\end{aligned}
$$


where $a, z$ and $R$ are functions of $r, r \in(0, \infty)$, and $s$ is the angular velocity. Just as in one spatial dimension, we can spatially discretize (54)-(56), find steady states of these equations, and continue them as parameters are varied, determining their stability with respect to radial perturbations as well. As example is shown in Fig. 12 (top), where $k$ is varied. The picture is qualitatively the same as in one dimension: the bump is destroyed in a saddle-node bifurcation as $k$ is decreased, and becomes unstable at $k \approx\left(\omega_{0}^{2}+4\right) / 2$, as the zero state becomes unstable.

As in one dimension, the saddle-node bifurcation in Fig. 12 (top) can be followed as another parameter, say $\omega_{0}$, is varied. The result is shown in Fig. 12 (bottom). Interestingly, the curve of saddle-node bifurcations terminates at a relatively high value of $\omega_{0}$ compared to the one-dimensional case (see Fig. 9). Although we have only considered perturbations in the radial direction, stability with respect to perturbations which break the rotational invariance of the solution could be performed in a straightforward manner [39, 40].

As an illustration that the analysis here predicts the behaviour of the discrete network, in Fig. 13 we show the results of a simulation of the two-dimensional version of $(2),(4),(5)$ where the order parameter $R$ is now defined as a double sum over all lattice points, and the coupling function is given by (52) rather than (1). A spatially-localised bump of synchronous activity surrounded by asynchronous oscillators is formed. This type of pattern is in some sense the opposite of the spiral wave chimeras seen in [14, 20,21], which have an asynchronous core surrounded by synchronous oscillators. Note that such spiral wave chimeras were found by [31] in (19),(20) and (53) at a variety of parameter values.

Interestingly, spherically-symmetric "bumps" of coherent oscillators surrounded by incoherent oscillators in three spatial dimensions will be described by (54)-(55) and

$$
\left(1-\frac{\partial^{2}}{\partial r^{2}}-\frac{2}{r} \frac{\partial}{\partial r}\right) R=\bar{a}
$$

and numerical simulation of these equations suggests that such solutions exist (not shown).

\section{Model 2: discrete and continuous}

The second model we consider is the spatially-extended version of a model recently introduced by Hong and Strogatz [32]. We define a non-local mean field, and each oscillator is directly coupled to this, but by a coupling strength that is randomly chosen to be one of two values, of differing sign. Those with positive coupling strength were labelled by Hong and Strogatz "conformists," while those with negative coupling strength were labelled "contrarians." For some parameter values, the non-spatial model of [32] was found to exhibit bistability, and it is this property that allows us to construct fronts in the spatially-extended system.

\subsection{Spatially discrete}

We again consider $N$ phase oscillators equally spaced on the one dimensional spatial domain $[0, L]$ and define the nonlocal mean field

$$
R_{k}(t)=\frac{L}{N} \sum_{j=1}^{N} K_{k j} e^{i \theta_{j}(t)}
$$

for $k=1, \ldots, N$, where

$$
K_{i j}=K(|i-j| \Delta x), \quad K(x)=(1 / 2) e^{-|x|}
$$

and $\Delta x=L /(N-1)$. Writing $R_{j}=\rho_{j} e^{i \phi_{j}}$ our dynamics are

$$
\frac{d \theta_{j}}{d t}=\omega_{j}+\sigma_{j} \rho_{j} \sin \left(\phi_{j}-\theta_{j}\right) \quad j=1, \ldots N
$$

where, as before, the $\omega_{j}$ are chosen from the distribution $g(\omega)$, and the $\sigma_{j}$ are chosen independently of the $\omega_{j}$ from the double- $\delta$ distribution

$$
\Gamma(\sigma) \equiv(1-p) \delta\left(\sigma-\sigma_{1}\right)+p \delta\left(\sigma-\sigma_{2}\right)
$$



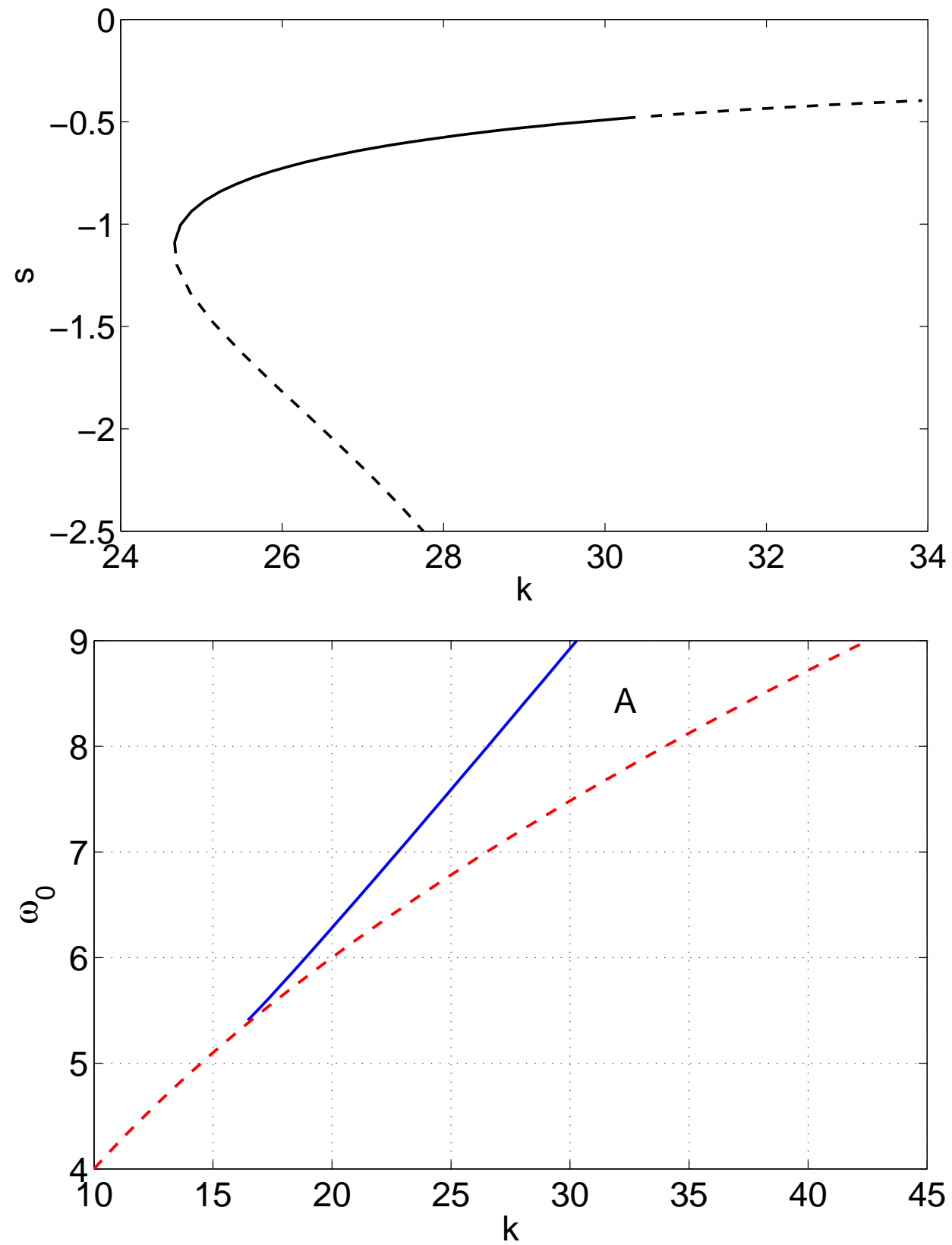

Figure 12: Top: Angular velocity $s$ for a stationary bump solution of (54)-(56), as a function of $k$. Solid line: stable with respect to radial perturbations; dashed: unstable with respect to radial perturbations. Other parameters: $\omega_{0}=7.5$. Bottom: Solid blue: saddle-node bifurcation of a 1-bump solution of (54)-(56). Red dashed line: $k=\left(\omega_{0}^{2}+4\right) / 2$, to the right of which the zero state is unstable. A circularly-symmetric bump, stable with respect to radial perturbations, exists only in region A. Compare with Fig. 9. 

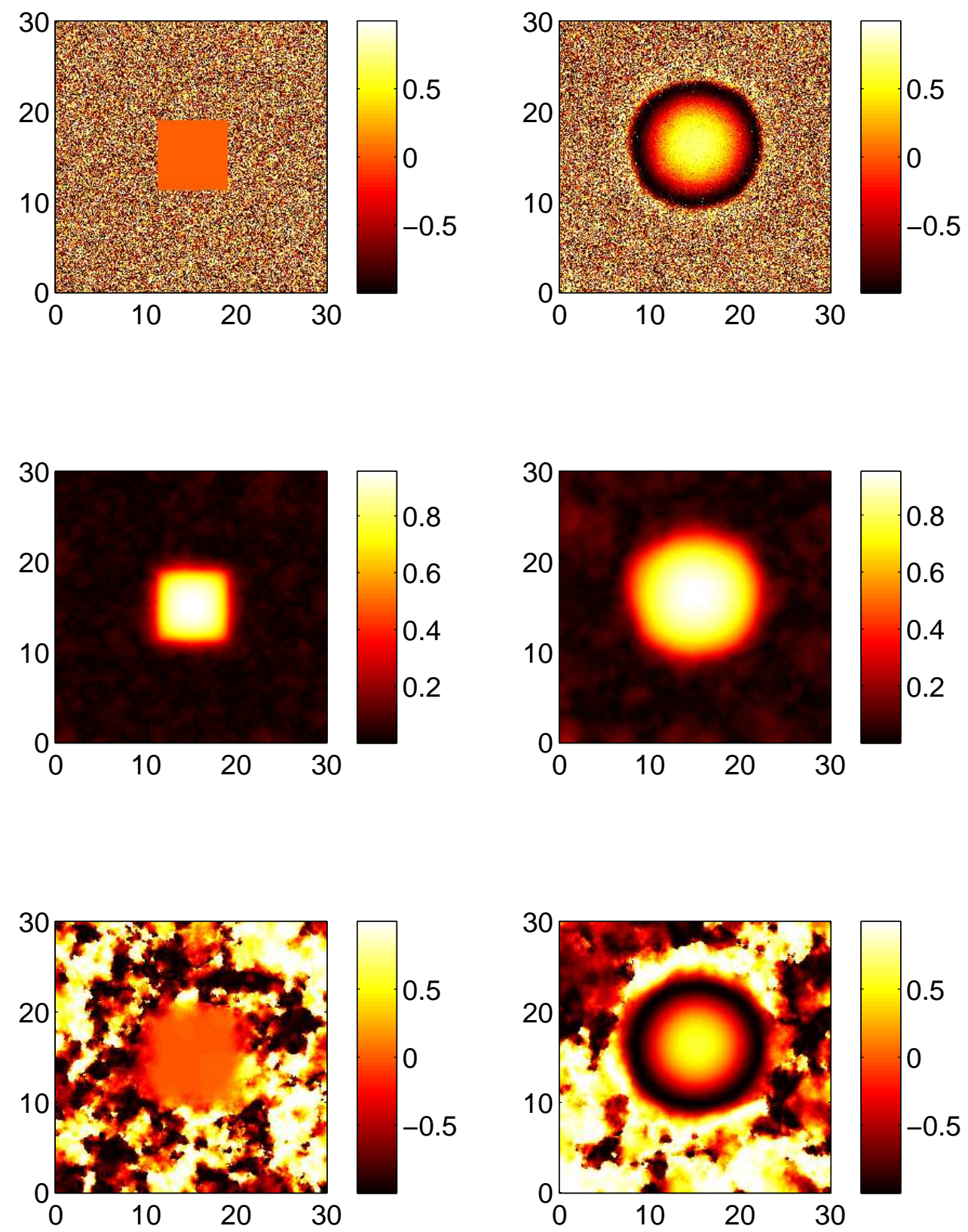

Figure 13: Snapshots of a simulation of the two-dimensional version of (2),(4),(5). Left column: $t=0$; right column: $t=200$ Top row: $\sin \left(\theta_{i j}\right)$; middle row: $\left|R_{i j}\right|$; bottom row: $\sin \left(\arg \left(R_{i j}\right)\right)$. Compare with Fig. 11 . The domain is $30 \times 30$ and $252 \times 252$ oscillators were used. Parameters: $\omega_{0}=8, k=30$. 

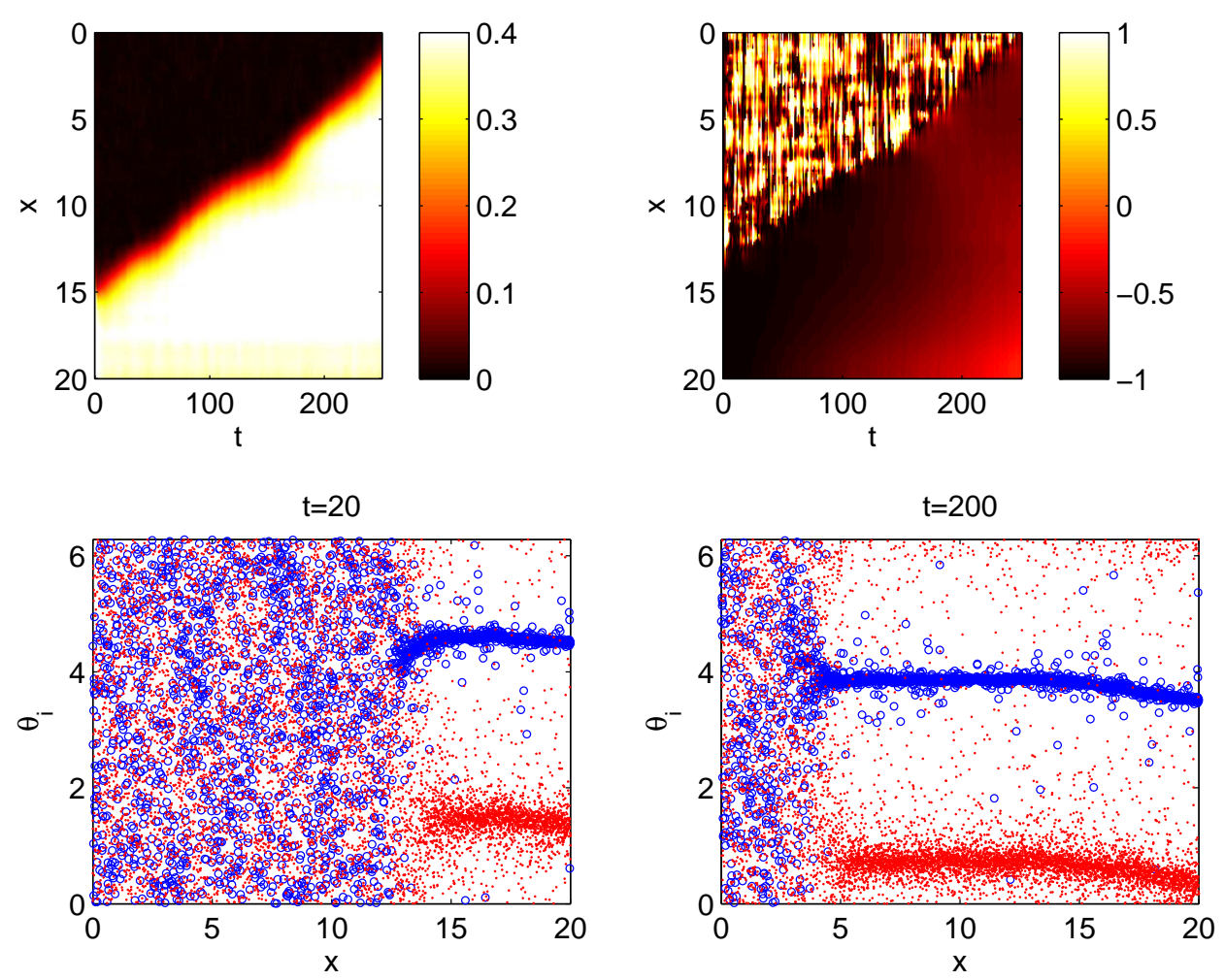

Figure 14: A typical solution of (58)-(60). Top left: $\rho_{j}(t)$; top right: $\sin \left(\phi_{j}(t)\right)$, where $R_{j}=\rho_{j} e^{i \phi_{j}}$. Bottom row: snapshots of the network at $t=20$ (left) and $t=200$ (right). Oscillators with $\sigma_{j}<0$ (contrarians) are shown with blue circles, and those with $\sigma_{j}>0$ (conformists) with red dots. Parameters: $\sigma_{1}=-10, \sigma_{2}=1, \gamma=0.05, p=0.75, N=10,000, L=20$.

where $\sigma_{1}<0$ and $\sigma_{2}>0$, and $p$ is a probability. An example of the typical dynamics, where

$$
g(\omega)=\frac{\gamma / \pi}{\omega^{2}+\gamma^{2}}
$$

is shown in Fig. 14. For initial conditions, we chose the phase of oscillators between $x=0$ and $x=15$ randomly from a uniform distribution on $[0,2 \pi]$, and those between $x=15$ and $x=20$ were all set at $\theta_{j}(0)=\pi / 2$. Very rapidly, the initially synchronous oscillators split into two groups - based on the sign of their coupling coefficient - with average phase differing by approximately $\pi$, as predicted by the analysis in [32]. A wave of partial synchrony then propagated across the domain, invading the region of complete asynchrony. One point to note is that, unlike Model 1, the phases of the synchronous oscillators do not seem to vary significantly over time.

\subsection{Continuum model}

The derivation of the continuum version of (58)-(60) is very similar to that in Sec. 2.2 and in [32], so we are brief. In the limit $N \rightarrow \infty$ the system is described by a probability density function $F(x, \omega, \theta, \sigma, t)$ which satisfies

$$
\frac{\partial F}{\partial t}+\frac{\partial}{\partial \theta}\{F(\omega+\sigma v)\}=0
$$

where

$$
v(x, \theta, t)=\int_{0}^{L} K(x-y) \int_{-\infty}^{\infty} \int_{-\pi}^{\pi} \int_{-\infty}^{\infty} \sin \left(\theta^{\prime}-\theta\right) F\left(y, \omega, \theta^{\prime}, \sigma^{\prime}, t\right) \Gamma\left(\sigma^{\prime}\right) d \sigma^{\prime} d \theta^{\prime} d \omega d y
$$


The non-local mean field (58) becomes

$$
R(x, t)=\int_{0}^{L} K(x-y) \int_{-\infty}^{\infty} \int_{-\pi}^{\pi} \int_{-\infty}^{\infty} e^{i \theta^{\prime}} F\left(y, \omega, \theta^{\prime}, \sigma^{\prime}, t\right) \Gamma\left(\sigma^{\prime}\right) d \sigma^{\prime} d \theta^{\prime} d \omega d y
$$

and so $v$ can be written

$$
v(x, \theta, t)=\frac{R(x, t) e^{-i \theta}-\bar{R}(x, t) e^{i \theta}}{2 i}
$$

Writing

$$
F(x, \omega, \theta, \sigma, t)=\frac{g(\omega)}{2 \pi}\left[1+\left(\sum_{n=1}^{\infty}\{\alpha(x, \omega, \sigma, t)\}^{n} e^{i n \theta}+\text { c.c. }\right)\right]
$$

we obtain

$$
R(x, t)=\int_{0}^{L} K(x-y) \int_{-\infty}^{\infty} \int_{-\infty}^{\infty} \bar{\alpha}(y, \omega, \sigma, t) g(\omega) \Gamma(\sigma) d \sigma d \omega d y
$$

and

$$
\frac{\partial \alpha(x, \omega, \sigma, t)}{\partial t}=-i \omega \alpha(x, \omega, \sigma, t)+(\sigma / 2)\left[\bar{R}(x, t)-R(x, t) \alpha^{2}(x, \omega, \sigma, t)\right]
$$

Using (62) we have

$$
R(x, t)=\int_{0}^{L} K(x-y) \int_{-\infty}^{\infty} \bar{\alpha}(y,-i \gamma, \sigma, t) \Gamma(\sigma) d \sigma d y
$$

and defining $a(x, \sigma, t)=\alpha(x,-i \gamma, \sigma, t)$ we have

$$
\frac{\partial a(x, \sigma, t)}{\partial t}=-\gamma a(x, \sigma, t)+(\sigma / 2)\left[\bar{R}(x, t)-R(x, t) a^{2}(x, \sigma, t)\right]
$$

Using (61) we have

$$
R(x, t)=\int_{0}^{L} K(x-y)\left[(1-p) \bar{r}_{1}(y, t)+p \bar{r}_{2}(y, t)\right] d y
$$

where $r_{i}(x, t)=a\left(x, \sigma_{i}, t\right)$ for $i=1,2$. Using (71), the $r_{i}$ evolve according to

$$
\begin{aligned}
& \frac{\partial r_{1}}{\partial t}=-\gamma r_{1}+\left(\sigma_{1} / 2\right)\left[\bar{R}-R r_{1}^{2}\right] \\
& \frac{\partial r_{2}}{\partial t}=-\gamma r_{2}+\left(\sigma_{2} / 2\right)\left[\bar{R}-R r_{2}^{2}\right]
\end{aligned}
$$

Equations (73)-(74), together with (72), form our dynamical system. Several points can be noted:

- In one spatial dimension, eqn. (72) can be written

$$
\left(1-\frac{\partial^{2}}{\partial x^{2}}\right) R(x, t)=(1-p) \bar{r}_{1}(x, t)+p \bar{r}_{2}(x, t)
$$

- The space $\left\{\left(r_{1}, r_{2}\right): r_{1} \in \mathbb{R}, r_{2} \in \mathbb{R}\right\}$ is invariant.

- Time can always be rescaled so that $\sigma_{2}=1$, and we will assume from now on that this has been done.

A solution of (72)-(74) for the same parameters as used in Fig. 14 is shown in Fig. 15 (real initial conditions were used). We see a travelling wave very similar to that in Fig. 14, top left. 

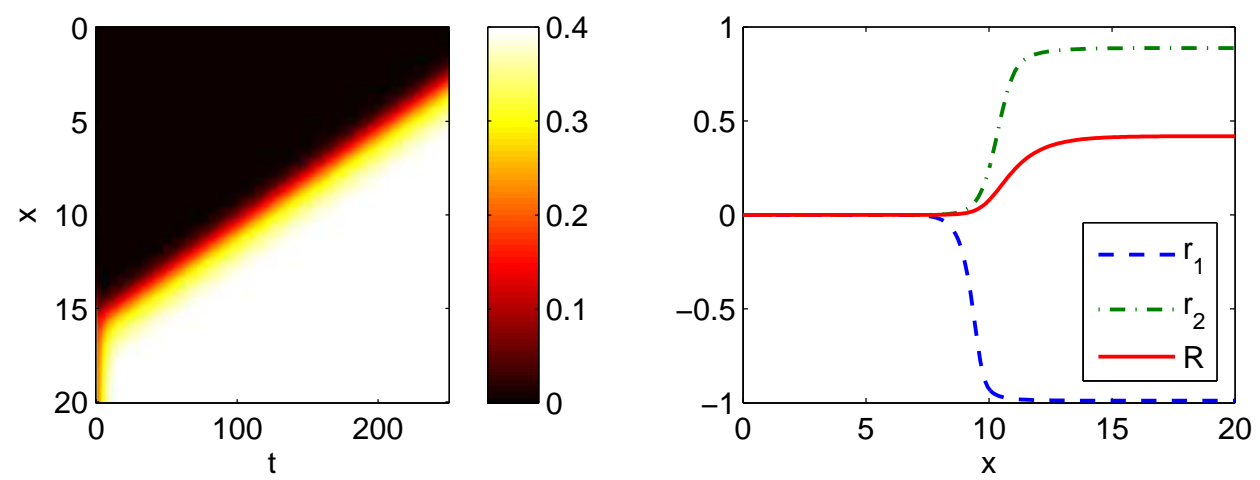

Figure 15: A solution of (72)-(74). Left: $R$ is shown color-coded (compare with top left panel of Fig. 14). Right: $r_{1}, r_{2}$ and $R$ at $t=100$. Parameters: $\sigma_{1}=-10, \sigma_{2}=1, \gamma=0.05, p=0.75, L=20$.

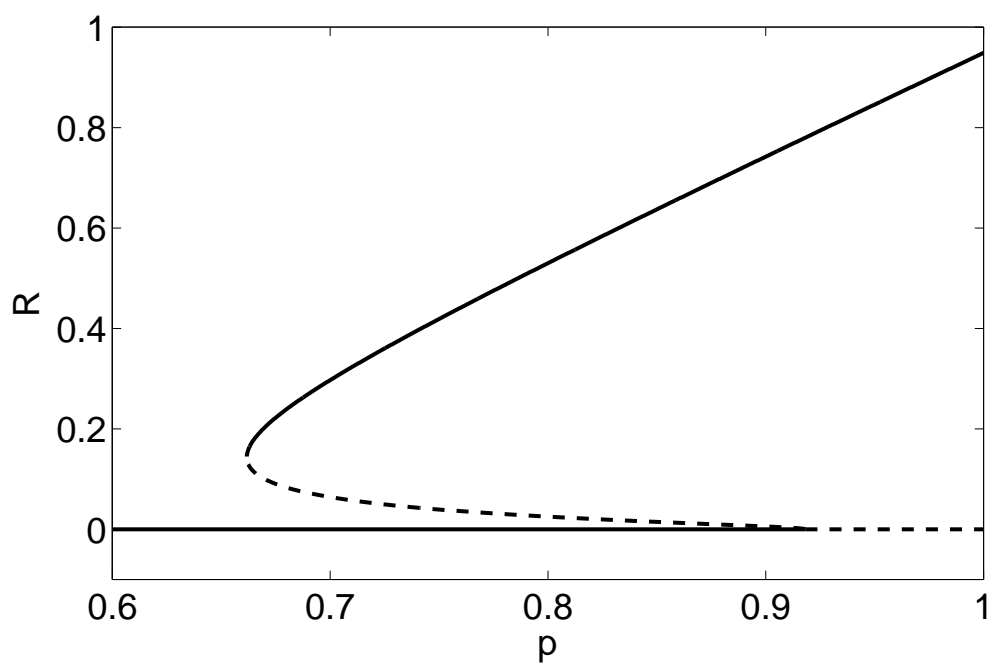

Figure 16: Spatially uniform steady states of (72)-(74) for which $r_{1}$ and $r_{2}$ are real and of opposite signs (" $\pi$-states"). Their stability is indicated: solid - stable; dashed - unstable. Parameters: $\sigma_{1}=-10, \sigma_{2}=1, \gamma=0.05$.

\section{Results}

Spatially-uniform states of (72)-(74) were studied in [32], where the authors found for $Q \equiv-\sigma_{1}>$ 0 large enough and $\gamma$ small enough the system displayed bistability over a range of values of $p$. In particular, for the parameter values we have used $(Q=10, \gamma=0.05)$ there exists a branch of " $\pi$-states" given by

$$
p=\frac{\gamma-\sqrt{\gamma^{2}+(Q R)^{2}}-Q R^{2}}{\gamma-\sqrt{\gamma^{2}+(Q R)^{2}}+\gamma Q-Q \sqrt{\gamma^{2}+R^{2}}}
$$

which we plot in Fig. 16, also indicating the stability of these states in the absence of spatial structure. In these $\pi$-states, $r_{1}$ and $r_{2}$ are real and of opposite signs (see Fig. 15 right panel). The branch of $\pi$-states terminates when $R=0$ at $p_{c}=(Q+2 \gamma) /(Q+1)$. A Turing analysis of the spatially uniform states, under the assumption that $r_{1}$ and $r_{2}$ are real, shows that the introduction of spatial structure does not lead to any new bifurcations of these states (results not shown). Rather than undertake a complete analysis of (72)-(74) here, we will study moving fronts like that in Fig. 15 under the restriction that $r_{1}$ and $r_{2}$ (and thus $R$ ) are real. 


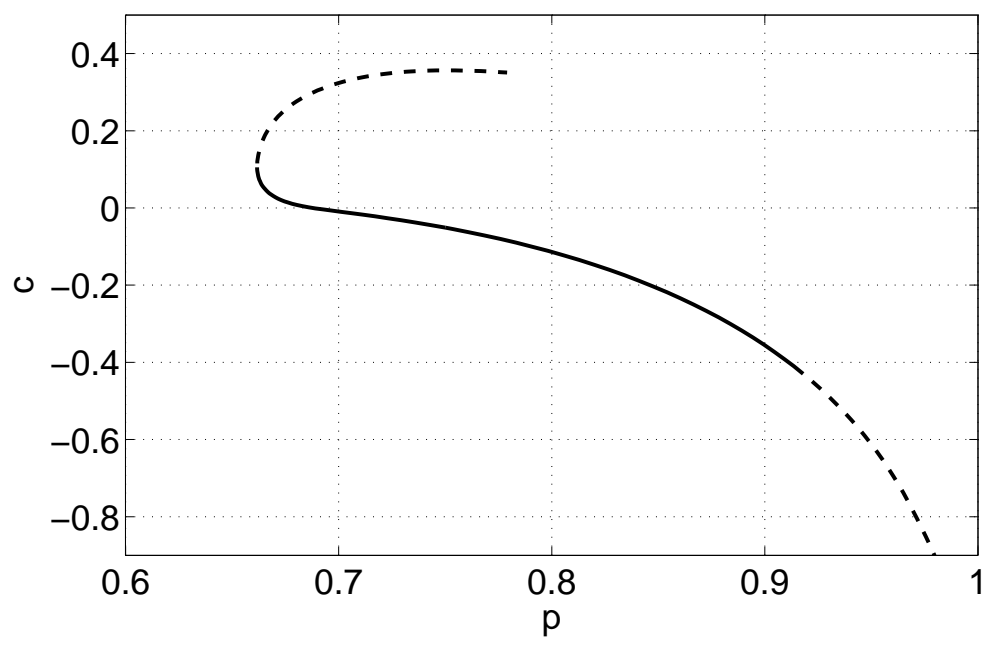

Figure 17: Speed $c$ of a front solution which is a fixed point of (77)-(79), as a function of $p$. Solid: stable; dashed: unstable. Parameters: $Q=10, \gamma=0.05$.

In a coordinate frame moving with speed $c$, and restricting the variables to be real, eqns. (73)-(75) become

$$
\begin{aligned}
\frac{\partial r_{1}}{\partial t} & =c \frac{\partial r_{1}}{\partial x}-\gamma r_{1}-(Q / 2)\left[R-R r_{1}^{2}\right] \\
\frac{\partial r_{2}}{\partial t} & =c \frac{\partial r_{2}}{\partial x}-\gamma r_{2}+(1 / 2)\left[R-R r_{2}^{2}\right] \\
\left(1-\frac{\partial^{2}}{\partial x^{2}}\right) R & =(1-p) r_{1}+p r_{2}
\end{aligned}
$$

As in Sec. 3.2, we can find steady states of (77)-(79) which satisfy a pinning condition of the form (32), i.e.

$$
\int_{0}^{L} \frac{d \widehat{u}}{d x}\left(r_{1}(x, t)-\widehat{u}\right) d x=0
$$

where $\widehat{u}(x)$ is a fixed template function, and follow them as, say, $p$ is varied. The result is shown in Fig. 17. As for Model 1, we obtain a stable branch of fronts over the interval for which the spatially uniform system shows bistability (see Fig. 16). The front is destroyed in a saddle-node bifurcation at the same parameter value at which the spatially uniform system shows this bifurcation, and the front becomes unstable as $p$ is increased through $p_{c}$, where the zero state loses stability. One interesting difference between Model 2 and Model 1, visible in Fig. 17, is that Model 2 is capable of supporting fronts which travel in both directions, as well as stationary fronts.

Following the saddle-node bifurcation shown in Fig. 17 as $\gamma$ is varied, we obtain the results shown in Fig. 18 (top), and varying $Q$ we obtain the results in Fig. 18 (bottom). As in Model 1, we obtain stable fronts only when the non-spatial system is bistable, between the blue solid and green dashed curves in Figs. 18.

In a similar way to Model 1, front solutions of (77)-(79) can be thought of as heteroclinic connections between the origin and the upper fixed point for the spatial ODEs obtained by setting the time 

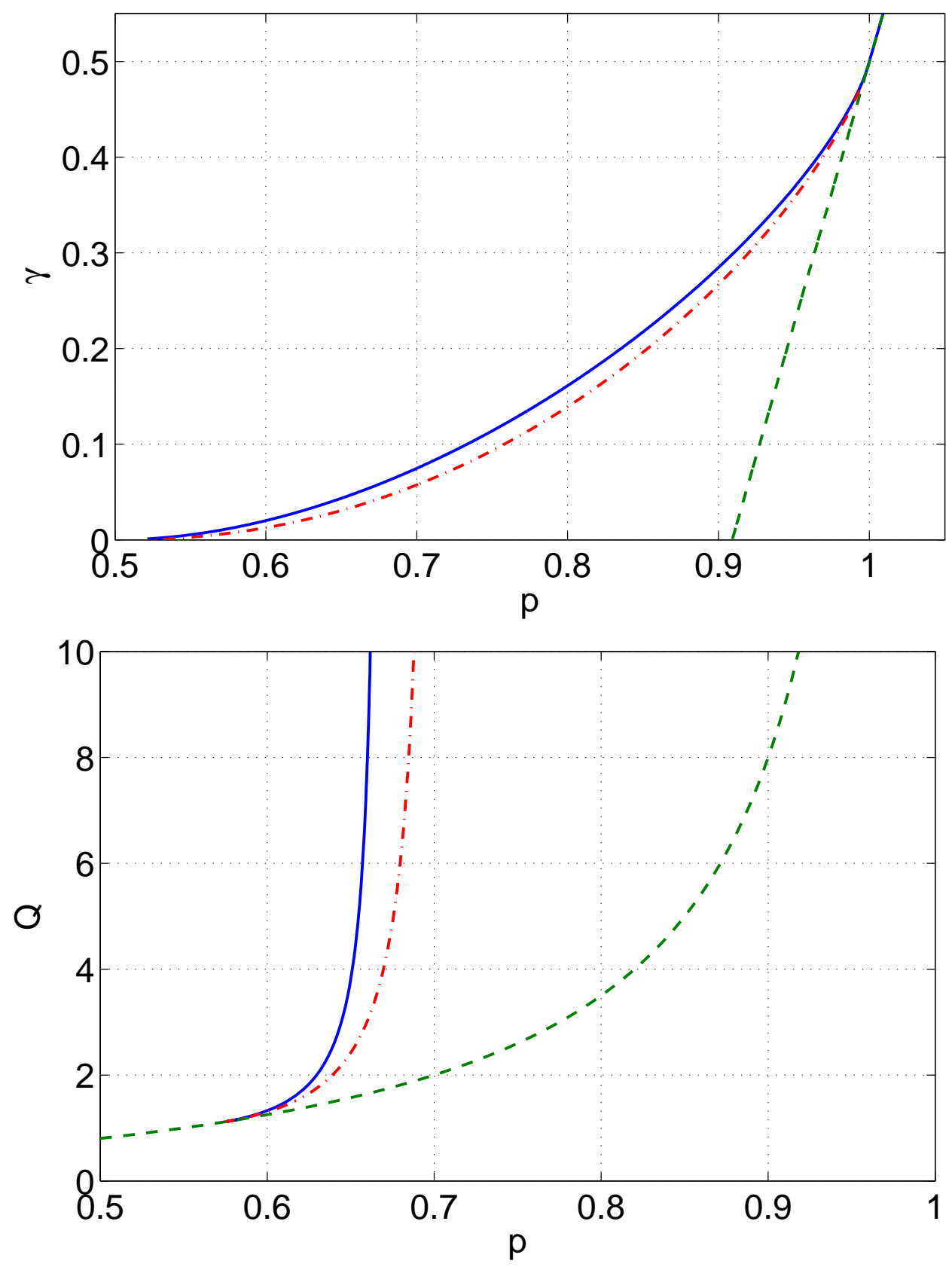

Figure 18: Solid blue: saddle-node bifurcation of front solutions of (73)-(75). Dashed green: $p_{c}$. Stable fronts exist only between the solid blue and green dashed lines. Red dash-dotted: the $c=0$ curve for stable fronts. Top: $Q=10$. Bottom: $\gamma=0.05$. 
derivatives in (77)-(78) to zero. For non-zero $c$ we obtain four first-order ODEs

$$
\begin{aligned}
\frac{d r_{1}}{d x} & =\left[\gamma r_{1}+(Q R / 2)\left(1-r_{1}^{2}\right)\right] / c \\
\frac{d r_{2}}{d x} & =\left[\gamma r_{2}-(R / 2)\left(1-r_{2}^{2}\right)\right] / c \\
\frac{d R}{d x} & =s \\
\frac{d s}{d x} & =R-\left[(1-p) r_{1}+p r_{2}\right]
\end{aligned}
$$

A special case occurs when $c=0$, for which these equations reduce to the planar system

$$
\begin{aligned}
& \frac{d R}{d x}=s \\
& \frac{d s}{d x}=R-\left[(1-p) r_{1}+p r_{2}\right]
\end{aligned}
$$

where

$$
r_{1}=\frac{\gamma-\sqrt{\gamma^{2}+(Q R)^{2}}}{Q R} \quad \text { and } \quad r_{2}=\frac{-\gamma+\sqrt{\gamma^{2}+R^{2}}}{R}
$$

or

$$
\frac{d^{2} R}{d x^{2}}-f(R)=0
$$

where $f(R)=(1-p) r_{1}+p r_{2}-R$. The vector field given by (85)-(86) is shown in Fig. 19 for three different values of $p$, along with the fixed points and part of the unstable manifold of the origin. We see that for only one value of $p$ is there a heteroclinic connection between the origin and a fixed point.

Solutions of (88) are given by

$$
\frac{s^{2}}{2}+F(R)=\text { const. }
$$

where

$$
F(R)=\int_{0}^{R} f(z) d z
$$

At $(R, s)=(0,0)$, the constant in (89) is zero and thus the heteroclinic connection between the origin and $\left(R^{*}, 0\right)$ - corresponding to a stationary front - can be found by solving the scalar equation

$$
F\left(R^{*}\right)=0
$$

where $R^{*}$ is the most positive root of $f(R)$. This situation is shown in Fig. 19 (b). A solution of (91) can be followed as other parameters are varied, giving the results shown with dash-dotted curves in Fig. 18.

As can be seen in Fig. 19 (c), for values of $p$ greater than the value at which the heteroclinic connection occurs there are codimension-zero homoclinic connections to the origin, representing stationary "bump" solutions. However, when substituted into (72)-(74) we found that these were unstable.

\section{Restriction to real solutions}

In this section we have analysed (72)-(74) under the restriction that $r_{1}$ and $r_{2}$ (and hence $R$ ) are real. From the analysis in [32] we know that such a restriction means that we will not capture all of the behaviour of the system for all parameter values (when $Q$ is small, for example). However, all of the results presented in this section have been reproduced using complex initial conditions, so - at least for the parameter values considered here - this restriction is not important. 

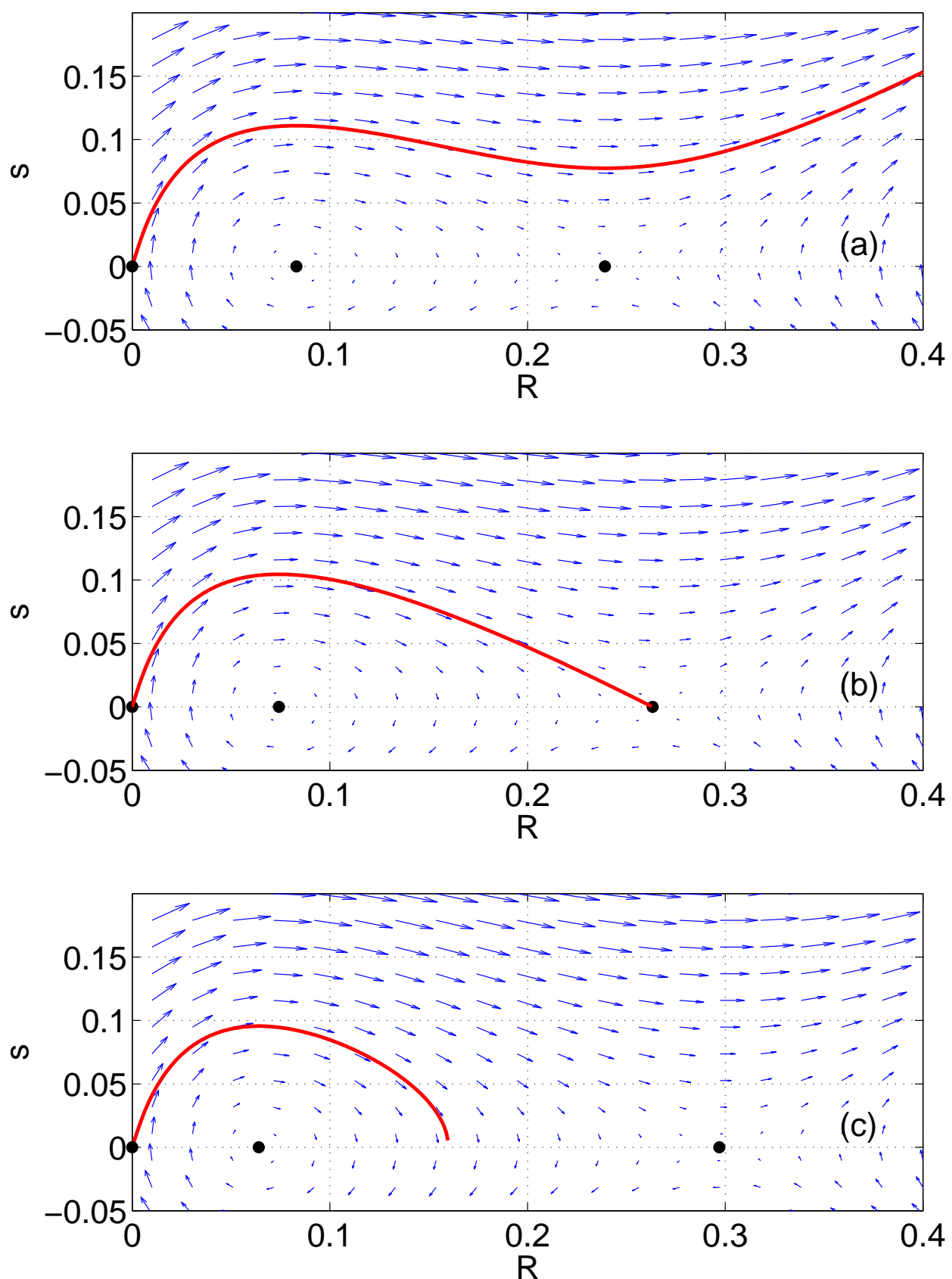

Figure 19: Vector fields of (85)-(86) (blue arrows) for $p=0.68$ (a); $p=0.68788$ (b); and $p=0.7$ (c). Fixed points are shown with black filled circles (the rightmost one has $R=R^{*}$ ). Part of the unstable manifold of the origin is shown with the thick red line. The heteroclinic connection between the origin and $\left(R^{*}, 0\right)$ occurs in panel (b). Other parameters: $Q=10, \gamma=0.05$. 


\section{Discussion}

In this paper we have analysed moving and stationary fronts and "bumps" in several different networks of phase oscillators coupled non-locally through phase differences. These fronts connect regions of high local synchrony with regions of complete asynchrony, and the bumps consist of spatiallylocalised regions of partially-synchronous behaviour. A crucial tool we have used is the Ott-Antonsen $(\mathrm{OA})$ ansatz $[8,30]$ which reduces the dynamics of such systems in the continuum limit to non-local differential equations [21]. Once these equations were derived, moving patterns were studied by either "freezing" them [33,34], or by regarding them as homoclinic or heteroclinic connections in a set of low-dimensional ordinary differential equations. The analysis of the continuum limit described well the behaviour seen in large finite networks of oscillators. Although moving fronts are necessarily transient, and thus do not describe the steady state of a network, their existence may well be useful in understanding observed transients, or the behaviour of a network with time-varying parameters.

For Model 1, previously derived by [31], we found regions of existence and stability for moving fronts in one spatial dimension and stationary bumps in one and two spatial dimensions. For Model 2, a generalisation of the model of [32], we found the regions of existence and stability for moving fronts in one spatial dimension. Interestingly, Model 2 supports fronts with positive, negative, or zero velocity, although not bumps.

A necessary ingredient for the existence of stable fronts is bistability in the network when spatial interactions are not present. We thus expect to find fronts in networks formed by non-locally coupling systems which have this bistability such as the periodically-forced Kuramoto model [41], the Kuramoto model with a bimodal frequency distribution [10], and the Kuramoto model with coupling strength inhomogeneity [11, 42].

We now discuss several generalisations of the models studied here. For both models we chose the distribution of intrinsic frequencies of the oscillators to be Lorentzian (eqns. (14) and (62)). This enabled us to perform the integral over intrinsic frequency explicitly, as has been done many times before [25, $21,10,3]$. Other distributions could be used, but for the OA ansatz to be valid the oscillators cannot have identical intrinsic frequencies [43]. On the other hand, if noise is added to the network, or higher harmonics are included in the phase coupling function, the OA ansatz can no longer be used.

For both models we used the spatial coupling function $K(x)=(1 / 2) e^{-|x|}$ in one dimension which, being the Green's function of $1-\partial^{2} / \partial x^{2}$, enabled us to invert the convolution integral involving $K$. This technique has been used before in, for example, neural field modelling [39, 40, 44, 45, 38]. For this coupling function neither model showed Turing bifurcations, i.e. instabilities of spatially-uniform states with respect to patterns with finite spatial wave-numbers. However, experience with neural field models suggests that non-monotonic coupling functions may well result in Turing bifurcations [46, 45], providing another route to spatio-temporal pattern formation in spatially-extended Kuramoto networks.

In much of the recent work on chimera states [20,21,22, 23, 24, 25,28], the presence of a phase offset in the term involving the sine of a phase difference was essential for the existence of such states. Neither of the models studied here had such a term but it would be straight-forward to include it, replacing $\sin \left(\phi_{j}-\theta_{j}\right)$ in (5) or (60) by $\sin \left(\phi_{j}-\theta_{j}-\alpha\right)$, where $\alpha$ is a parameter. This parameter then appears in the continuum description, and varying it may result in more interesting behaviour than that shown here. In a similar vein, if the distribution of $\sigma$ in Model 2 was a double Lorentzian rather than a double- $\delta$ distribution, $\sigma_{1}$ and $\sigma_{2}$ in (73) and (74) would be replaced by $\sigma_{1}+i \Delta_{1}$ and $\sigma_{2}+i \Delta_{2}$ respectively, where $\Delta_{1}$ and $\Delta_{2}$ are the half-width-at-half-maxima of the two distributions. Including these parameters may well result in new phenomena.

Another intriguing idea is that if some sort of slow feedback, driven by the degree of local synchrony, was included [47], it may be possible to obtain a moving bump, i.e. a spatially-localised region of partly synchronous oscillators moving through a background of asynchrony at a constant speed.

In summary, we have shown how to analyse a variety of spatio-temporal patterns that occur in networks of non-locally coupled, non-identical, phase oscillators. Our analysis of the equations describing the continuum limit is not complete, and there may well be more interesting behaviour to discover in these networks. 
Acknowledgements: I thank Alan Champneys for helpful conversations regarding this work and the referees for their useful comments.

\section{References}

[1] A. Pikovsky, M. Rosenblum, and J. Kurths. Synchronization. Cambridge University Press, 2001.

[2] S. Strogatz. Sync: The Emerging Science of Spontaneous Order. Hyperion, 2003.

[3] Yoshiki Kuramoto. Chemical oscillations, waves, and turbulence. Springer-Verlag, 1984.

[4] S.H. Strogatz. From Kuramoto to Crawford: exploring the onset of synchronization in populations of coupled oscillators. Physica D, 143:1-20, 2000.

[5] J.A. Acebrón, LL Bonilla, C.J. Pérez Vicente, F. Ritort, and R. Spigler. The Kuramoto model: A simple paradigm for synchronization phenomena. Rev. Mod. Phys., 77:137-185, 2005.

[6] Arkady Pikovsky and Michael Rosenblum. Partially integrable dynamics of hierarchical populations of coupled oscillators. Phys. Rev. Lett., 101:264103, 2008.

[7] S.A. Marvel, R.E. Mirollo, and S.H. Strogatz. Identical phase oscillators with global sinusoidal coupling evolve by Möbius group action. Chaos, 19:043104, 2009.

[8] Edward Ott and Thomas M. Antonsen. Low dimensional behavior of large systems of globally coupled oscillators. Chaos, 18:037113, 2008.

[9] Daniel M. Abrams, Rennie Mirollo, Steven H. Strogatz, and Daniel A. Wiley. Solvable model for chimera states of coupled oscillators. Phys. Rev. Lett., 101:084103, 2008.

[10] E. A. Martens, E. Barreto, S. H. Strogatz, E. Ott, P. So, and T. M. Antonsen. Exact results for the Kuramoto model with a bimodal frequency distribution. Physical Review E, 79:026204, 2009.

[11] T.W. Ko and G.B. Ermentrout. Partially locked states in coupled oscillators due to inhomogeneous coupling. Physical Review E, 78(1):016203, 2008.

[12] L. Ren and B. Ermentrout. Monotonicity of phaselocked solutions in chains and arrays of nearest-neighbor coupled oscillators. SIAM journal on mathematical analysis, 29(1):208-234, 1998.

[13] H. Hong, Hyunggyu Park, and M. Y. Choi. Collective synchronization in spatially extended systems of coupled oscillators with random frequencies. Phys. Rev. E, 72(3):036217, Sep 2005.

[14] S. Shima and Y. Kuramoto. Rotating spiral waves with phase-randomized core in nonlocally coupled oscillators. Physical Review E, 69(3):036213, 2004.

[15] C.R. Laing and C.C. Chow. Stationary Bumps in Networks of Spiking Neurons. Neural Comput., 13:1473-1494, 2001.

[16] J. Rinzel, D. Terman, X.J. Wang, and B. Ermentrout. Propagating activity patterns in large-scale inhibitory neuronal networks. Science, 279(5355):1351, 1998.

[17] Y. Kuramoto and D. Battogtokh. Coexistence of Coherence and Incoherence in Nonlocally Coupled Phase Oscillators. Nonlinear Phenom. Complex Syst., 5:380-385, 2002.

[18] P.J. Kim, T.W. Ko, H. Jeong, and H.T. Moon. Pattern formation in a two-dimensional array of oscillators with phase-shifted coupling. Physical Review E, 70(6):065201, 2004.

[19] Oleh E. Omel'chenko, Matthias Wolfrum, and Yuri L. Maistrenko. Chimera states as chaotic spatiotemporal patterns. Phys. Rev. E, 81(6):065201, Jun 2010.

[20] E.A. Martens, C.R. Laing, and S.H. Strogatz. Solvable model of spiral wave chimeras. Physical review letters, 104(4):044101, 2010.

[21] C. Laing. The dynamics of chimera states in heterogeneous Kuramoto networks. Physica D, 238:1569-1588, 2009.

[22] Daniel M. Abrams and Steven H. Strogatz. Chimera states for coupled oscillators. Phys. Rev. Lett., 93:174102, 2004.

[23] Daniel M. Abrams and Steven H. Strogatz. Chimera states in a ring of nonlocally coupled oscillators. Int. J. Bifn. Chaos, 16:21-37, 2006.

[24] G.C. Sethia, A. Sen, and F.M. Atay. Clustered chimera states in delay-coupled oscillator systems. Phys. Rev. Lett., 100:144102, 2008.

[25] Carlo R. Laing. Chimera states in heterogeneous networks. Chaos, 19:013113, 2009.

[26] Y. Kawamura. Chimera Ising walls in forced nonlocally coupled oscillators. Physical Review E, 75(5):056204, 2007.

[27] G. Bordyugov, A. Pikovsky, and M. Rosenblum. Self-emerging and turbulent chimeras in oscillator chains. Physical Review E, 82(3):035205, 2010.

[28] M. Wolfrum, O. E. Omel'chenko, S. Yanchuk, and Y. L. Maistrenko. Spectral properties of chimera states. Chaos, 21(1):013112, 2011.

[29] O.E. Omel'chenko, Y.L. Maistrenko, and P.A. Tass. Chimera States: The Natural Link Between Coherence and Incoherence. Phys. Rev. Lett., 100:044105, 2008.

[30] E. Ott and T.M. Antonsen. Long time evolution of phase oscillator systems. Chaos, 19:023117, 2009.

[31] Wai Shing Lee, Juan G. Restrepo, Edward Ott, and Thomas M. Antonsen. Dynamics and pattern formation in large systems of spatially-coupled oscillators with finite response times. Chaos, 21(2):023122, 2011.

[32] Hyunsuk Hong and Steven H. Strogatz. Kuramoto model of coupled oscillators with positive and negative coupling parameters: An example of conformist and contrarian oscillators. Phys. Rev. Lett., 106(5):054102, Feb 2011.

[33] W.J. Beyn and V. Thümmler. Freezing solutions of equivariant evolution equations. SIAM Journal on Applied Dynamical Systems, 3(2):85-116, 2004.

[34] G.J. Lord and V. Thümmler. Freezing Stochastic Travelling Waves. Arxiv preprint arXiv:1006.0428, 2010.

[35] W.S. Lee, E. Ott, and T.M. Antonsen. Large coupled oscillator systems with heterogeneous interaction delays. Physical review letters, 103(4):44101, 2009. 
[36] AR Champneys. Homoclinic orbits in reversible systems and their applications in mechanics, fluids and optics. Physica D: Nonlinear Phenomena, 112(1-2):158-186, 1998.

[37] Margaret Beck, Juergen Knobloch, David J. B. Lloyd, Bjoern Sandstede, and Thomas Wagenknecht. Snakes, ladders, and isolas of localized patterns. SIAM J MATH. ANAL., 41(3):936-972, 2009.

[38] C.R. Laing, W.C. Troy, B. Gutkin, and G.B. Ermentrout. Multiple bumps in a neuronal model of working memory. SIAM Journal on Applied Mathematics, 63:62, 2002.

[39] C.R. Laing and W.C. Troy. PDE methods for nonlocal models. SIAM J. Appl. Dyn. Syst, 2(3):487-516, 2003.

[40] MR Owen, CR Laing, and S. Coombes. Bumps and rings in a two-dimensional neural field: splitting and rotational instabilities. New Journal of Physics, 9:378, 2007.

[41] Lauren M. Childs and Steven H. Strogatz. Stability diagram for the forced Kuramoto model. Chaos, 18(4):043128, 2008

[42] Tae-Wook Ko and G. Bard Ermentrout. Bistability between synchrony and incoherence in limit-cycle oscillators with coupling strength inhomogeneity. Phys. Rev. E, 78(2):026210, Aug 2008.

[43] Edward Ott, Brian R. Hunt, and Jr. Thomas M. Antonsen. Comment on "Long time evolution of phase oscillator systems" [Chaos 19, 023117 (2009)]. Chaos, 21(2):025112, 2011

[44] C.R. Laing. Spiral waves in nonlocal equations. SIAM Journal on Applied Dynamical Systems, 4(3):588-606, 2005.

[45] S. Coombes, N.A. Venkov, L. Shiau, I. Bojak, D.T.J. Liley, and C.R. Laing. Modeling electrocortical activity through improved local approximations of integral neural field equations. Physical Review E, 76(5):051901, 2007.

[46] AJ Elvin, CR Laing, and MG Roberts. Transient Turing patterns in a neural field model. Physical Review E, 79(1):011911, 2009

[47] D. Taylor, E. Ott, and J.G. Restrepo. Spontaneous synchronization of coupled oscillator systems with frequency adaptation. Physical Review E, 81(4):046214, 2010. 